\title{
Lithium and zirconium abundances in massive Galactic O-rich AGB stars ${ }^{\star} \star \star$
}

\author{
D. A. García-Hernández ${ }^{1}$, P. García-Lario ${ }^{1,2}$, B. Plez ${ }^{3}$, A. Manchado ${ }^{4,5}$, F. D’Antona ${ }^{6}$, J. Lub ${ }^{7}$, and H. Habing ${ }^{7}$ \\ 1 ISO Data Centre, Research and Scientific Support Department of ESA. European Space Astronomy Centre (ESAC), \\ Villafranca del Castillo, PO Box 50727, 28080 Madrid, Spain \\ e-mail: agarcia@astro.as.utexas.edu \\ ${ }^{2}$ Herschel Science Centre, Research and Scientific Support Department of ESA. European Space Astronomy Centre (ESAC), \\ Villafranca del Castillo, PO Box 50727, 28080 Madrid, Spain \\ e-mail: Pedro.Garcia-Lario@sciops.esa.int \\ 3 GRAAL, CNRS UMR 5024, Université de Montpellier 2, 34095 Montpellier Cedex 5, France \\ e-mail: Bertrand.Plez@graal.univ-montp2.fr \\ 4 Instituto de Astrofísica de Canarias, La Laguna 38200, Tenerife, Spain \\ e-mail: amt@iac.es \\ ${ }_{5}$ Consejo Superior de Investigaciones Científicas (CSIC), Spain \\ ${ }^{6}$ Osservatorio Astronomico di Roma, via Frascati 33, 00040 MontePorzio Catone, Italy \\ e-mail: dantona@mporzio.astro.it \\ 7 Sterrewacht Leiden, Niels Bohrweg 2, 2333 RA Leiden, The Netherlands
}

Received 8 June 2006 / Accepted 25 August 2006

\begin{abstract}
Lithium and zirconium abundances (the latter taken as representative of s-process enrichment) are determined for a large sample of massive Galactic O-rich AGB stars, for which high-resolution optical spectroscopy has been obtained $(R \sim 40000-50000)$. This was done by computing synthetic spectra based on classical hydrostatic model atmospheres for cool stars and using extensive line lists. The results are discussed in the framework of "hot bottom burning" (HBB) and nucleosynthesis models. The complete sample is studied for various observational properties such as the position of the stars in the IRAS two-colour diagram ([12] - [25] vs. [25] - [60]), Galactic distribution, expansion velocity (derived from the $\mathrm{OH}$ maser emission), and period of variability (when available). We conclude that a considerable fraction of these sources are actually massive AGB stars $\left(M>3-4 M_{\odot}\right)$ experiencing $\mathrm{HBB}$, as deduced from the strong $\mathrm{Li}$ overabundances we found. A comparison of our results with similar studies carried out in the past for the Magellanic Clouds (MCs) reveals that, in contrast to MC AGB stars, our Galactic sample does not show any indication of s-process element enrichment. The differences observed are explained as a consequence of metallicity effects. Finally, we discuss the results obtained in the framework of stellar evolution by comparing our results with the data available in the literature for Galactic post-AGB stars and PNe.
\end{abstract}

Key words. stars: AGB and post-AGB - stars: abundances - stars: evolution - nuclear reactions, nucleosynthesis, abundances stars: atmospheres - stars: late-type

\section{Introduction}

The asymptotic giant branch (AGB) is formed by stars with initial masses in the range between 0.8 and $8 M_{\odot}$ in a late stage of their evolution. The internal structure of an AGB star consists of an electron-degenerate $\mathrm{C}-\mathrm{O}$ core surrounded by a shell of He. During most of the time $\mathrm{H}$ burning is the main source of energy for the AGB star but, occasionally, the inner He shell ignites in a "thermal pulse"; and eventually, the byproducts of He burning may reach the outer layers of the atmosphere in a "dredge-up" of processed material (the so-called third dredgeup). Repeated thermal pulses add carbon to the stellar surface. As a consequence, originally O-rich AGB stars can turn into

\footnotetext{
^ Based on observations at the $4.2 \mathrm{~m}$ William Herschel Telescope operated on the island of La Palma by the Isaac Newton Group in the Spanish Observatorio del Roque de Los Muchachos of the Instituto de Astrofisica de Canarias. Also based on observations with the ESO $3.6 \mathrm{~m}$ telescope at La Silla Observatory (Chile).

$\star \star$ Tables 1-9 are only available in electronic form at http://www. aanda.org
}

C-rich AGB stars $(\mathrm{C} / \mathrm{O}>1)$. This scenario would explain the observed spectral sequence M-MS-S-SC-C in AGB stars (e.g. Mowlavi 1999).

Another important characteristic of AGB stars is the presence of neutron-rich elements (s-elements such as $\mathrm{Sr}, \mathrm{Y}, \mathrm{Zr}$, $\mathrm{Ba}, \mathrm{La}, \mathrm{Nd}, \mathrm{Tc}$, etc.) in their atmospheres. These species are formed by slow-neutron captures in the intershell region. After a dredge-up episode, according to theoretical models, protons can be partially mixed in the ${ }^{12} \mathrm{C}$ - and ${ }^{4} \mathrm{He}$-rich region between the hydrogen- and helium-burning shells (e.g. Straniero et al. 1995, 1997; Mowlavi 2002; Lattanzio 2003) and can react with ${ }^{12} \mathrm{C}$ to form ${ }^{13} \mathrm{C}$ during the interpulse phase. The ${ }^{13} \mathrm{C}(\alpha, \mathrm{n}){ }^{16} \mathrm{O}$ reaction releases neutrons, which are captured by iron nuclei and other heavy elements, forming s-elements that can later be dredged up to the stellar surface in the next thermal pulse (Straniero et al. 1995, 1997; Busso et al. 2001).

Another neutron source, ${ }^{22} \mathrm{Ne}$, needs to be considered. In this case the activation takes place during the convective thermal pulses (e.g. Straniero et al. 1995, 2000; Vaglio et al. 1999; Gallino et al. 2000). ${ }^{22} \mathrm{Ne}$ can be formed from ${ }^{14} \mathrm{~N}$, 
and ${ }^{14} \mathrm{~N}$ is formed by the CNO cycle in the hydrogen shell. The ${ }^{22} \mathrm{Ne}(\alpha, \mathrm{n}){ }^{25} \mathrm{Mg}$ neutron source requires higher temperatures and typically occurs at higher neutron densities than the ${ }^{13} \mathrm{C}(\alpha, n){ }^{16} \mathrm{O}$ reaction. Thus, a different s-element pattern is expected depending on the dominant neutron source. According to the most recent models, the ${ }^{13} \mathrm{C}(\alpha, \mathrm{n}){ }^{16} \mathrm{O}$ reaction is the preferred neutron source for masses around 1-3 $M_{\odot}$, while for more massive stars (i.e. $M \gtrsim 3-4 M_{\odot}$ ) neutrons are thought to be mainly released through the ${ }^{22} \mathrm{Ne}(\alpha, \mathrm{n})^{25} \mathrm{Mg}$ reaction (see, for example, Busso et al. 1999 and Lattanzio \& Lugaro 2005, for a recent review). In the literature, there is strong evidence that most Galactic AGB stars enriched in s-process elements have masses around $1-3 M_{\odot}$, where the ${ }^{13} \mathrm{C}(\alpha, n){ }^{16} \mathrm{O}$ reaction is the neutron donor (e.g. Lambert et al. 1995; Abia et al. 2001). Unfortunately, a confrontation of the predictions made by these models with observations of more massive AGB stars in our Galaxy is not yet available.

In the case of the more massive O-rich AGB stars $(M>$ 3-4 $M_{\odot}$ ), the convective envelope can penetrate the H-burning shell thereby activating so-called "hot bottom burning" (hereafter, HBB), which takes place when the temperature at the base of the convective envelope is hot enough $(T \geq 2 \times$ $10^{7} \mathrm{~K}$ ) and ${ }^{12} \mathrm{C}$ can be converted into ${ }^{13} \mathrm{C}$ and ${ }^{14} \mathrm{~N}$ through the CN cycle (Sackmann \& Boothroyd 1992; Wood et al. 1983). The HBB models (Sackmann \& Boothroyd 1992; D'Antona \& Mazzitelli 1996; Mazzitelli et al. 1999) also predict the production of the short-lived ${ }^{7} \mathrm{Li}$ by the chain ${ }^{3} \mathrm{He}(\alpha, \gamma){ }^{7} \mathrm{Be}\left(\mathrm{e}^{-}, v\right)^{7} \mathrm{Li}$, through the so-called "7 Be transport mechanism" (Cameron \& Fowler 1971). One of the predictions of these models is that lithium should be detectable, at least for some time, on the stellar surface.

Activation of HBB in massive O-rich AGB stars is supported by studies of AGB stars in the Magellanic Clouds (hereafter, MCs) (Plez et al. 1993; Smith \& Lambert 1989, 1990a; Smith et al. 1995), which show a lack of high-luminosity $C$ stars beyond $M_{\text {bol }} \sim-6$. Instead, the more luminous AGB stars in the $\mathrm{MCs}$ are O-rich. Detection of strong Li overabundances, together with strong s-element enhancement in these luminous AGB stars in the LMC and in the SMC, is the signature that these stars are indeed HBB AGB stars that have undergone a series of thermal pulses and dredge-up episodes in their recent past. The HBB nature of these stars is also confirmed by the detection of a very small ${ }^{12} \mathrm{C} /{ }^{13} \mathrm{C}$ ratio $(\simeq 3-4)$, expected only when $\mathrm{HBB}$ is active (Mazzitelli et al. 1999). Unfortunately, current HBB theoretical models have been tested almost exclusively using the results of studying the more massive AGB stars in the MCs but have never been applied to Galactic sources, mainly because of the lack of observations available but also because of inaccurate information on distances within our Galaxy.

Indeed, only a handful of Li-rich stars have been found in our Galaxy so far (e.g. Abia et al. 1991, 1993; Boffin et al. 1993) and, unlike those detected in the MCs, they are not very luminous $\left(-6 \leq M_{\text {bol }} \leq-3.5\right)$. Most of them are low-mass C-rich AGB stars (Abia \& Isern 1996, 1997, 2000) and intermediatemass S- and SC-stars (Abia \& Wallerstein 1998) and not O-rich M-type stars. A few are less luminous red giant branch (RGB) stars at the bump (Charbonnel 2005). Some of these AGB stars are among the most Li-rich stars in our Galaxy, the so-called "super Li-rich" AGB stars (Abia et al. 1991). Under these conditions, however, HBB is not expected to be active and the Li production is not well-understood. Note that HBB is expected to be active only in the most massive (and luminous) AGB stars (from $\sim 4$ to $7 M_{\odot}$ ) (Mazzitelli et al. 1999), which should not be C-rich, but O-rich. Actually, the best candidates in our Galaxy are the so-called OH/IR stars, luminous O-rich AGB stars that are extremely bright in the infrared, showing a characteristic double-peaked $\mathrm{OH}$ maser emission at $1612 \mathrm{MHz}$. These stars are also known to be very long period variables (LPVs), sometimes with periods of more than 500 days and large amplitudes of up to 2 bolometric magnitudes. However, they experience very strong mass loss rates (up to several times $10^{-5} M_{\odot} \mathrm{yr}^{-1}$ ) at the end of the AGB, and most of them appear heavily obscured by thick circumstellar envelopes, making optical observations very difficult (e.g. Kastner et al. 1993; Jiménez-Esteban et al. 2005a, 2006a). Thus, no information exists yet on their lithium abundances and/or possible s-process element enrichment.

In this paper we present results from a wide observational programme based on high-resolution optical spectroscopy of a carefully selected sample of Galactic AGB stars thought to be massive from their observational properties. The criteria followed to select the sources in the sample are explained in Sect. 2. The high-resolution spectroscopic observations made in the optical and the data reduction process are described in Sect. 3, while the main results are presented in Sect. 4. In this section we also show how spectral synthesis techniques were applied to derive the atmospheric parameters, as well as the lithium and zirconium abundances, of the stars in our sample. A discussion of these results in the context of HBB models, nucleosynthesis models, and stellar evolution can be found in Sect. 5. Finally, the conclusions derived from this work are given in Sect. 6.

\section{Selection of the sample}

A large sample of long period (300-1000 days), large amplitude variability (up to 8-10 mag in the $V$ band), late-type (>M5) O-rich AGB stars displaying $\mathrm{OH}$ maser emission with a wide range of expansion velocities (from just a few $\mathrm{km} \mathrm{s}^{-1}$ to more than $20 \mathrm{~km} \mathrm{~s}^{-1}$ ) was carefully selected from the literature. These sources are all very bright in the infrared and, as such, included in the IRAS Point Source Catalogue (Beichman et al. 1988). Some of them are so strongly reddened that they do not show any optical counterpart in the Digitized Sky Survey ${ }^{1}$ (DSS) plates, although in a few cases the detection of these sources in the optical at a different epoch has been reported in the literature.

Stars were included in the sample if they satisfied at least one of the above conditions (ideally as many of them as possible), which guarantees that they are actually relatively massive stars. In Table 1 we list the 102 stars selected for analysis, together with their IRAS names and other being names from the literature, the Galactic coordinates, the variability type taken from the Combined General Catalogue of Variable Stars (GCVS, Kholopov 1998), the spectral type taken from Kwok et al. (1997) and references therein, and the IRAS colour indices [12] $-[25]$ and [25] $-[60]^{2}$. Similar information for a few well-known Galactic M-supergiants and S-, SC-, and C-type AGB stars that were also observed for comparison purposes is presented in Table 2. In addition, nine stars classified as Mira-type stars in SIMBAD ${ }^{3}$ and included in the initial sample were found to exhibit observational properties corresponding to C-rich stars. These are listed in Table 3.

\footnotetext{
1 The Digitized Sky Survey was produced at the Space Telescope Science Institute under US Government grant NAG W-2166.

2 Defined as [12] - [25] $=-2.5 \log \left(F_{12} / F_{25}\right)$ and [25]-[60] $=-2.5$ $\log \left(F_{25} / F_{60}\right)$, where $F_{12}, F_{25}$ and $F_{60}$ are the IRAS flux densities at 12 , 25 and $60 \mu \mathrm{m}$, respectively.

3 Set of Identifications, Measurements, and Bibliography for Astronomical Data, operated at CDS, Strasbourg, France.
} 


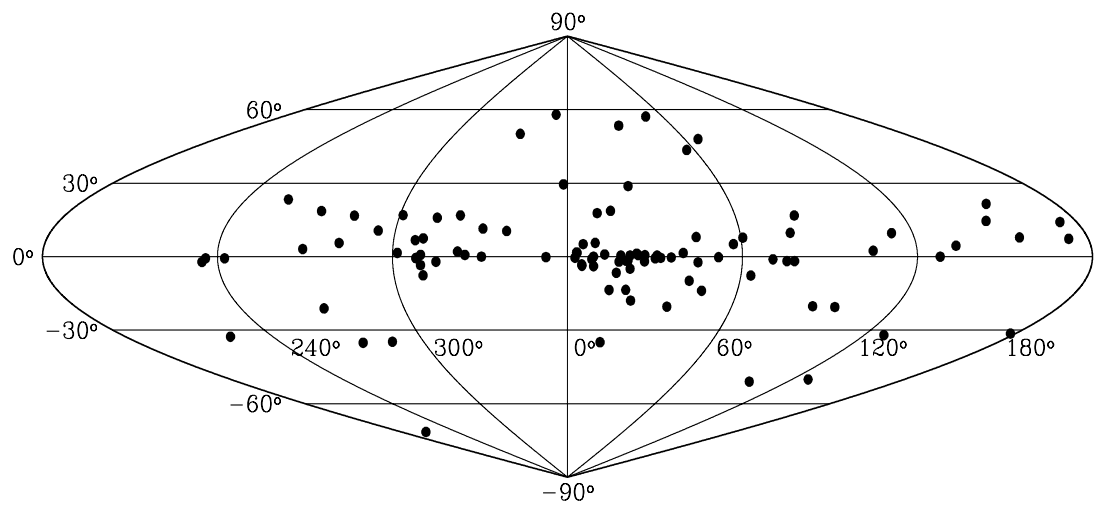

Fig. 1. Galactic distribution of the sources in the sample.

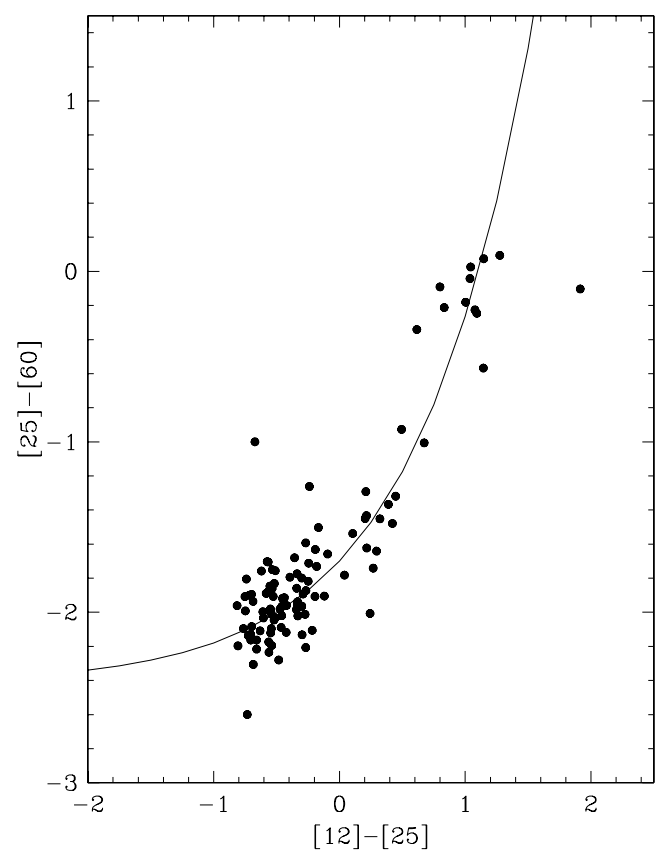

Fig. 2. IRAS two-colour diagram [12] - [25] vs. [25] - [60], where the positions of all the sources in the sample are represented (black dots). The continuous line is the "O-rich AGB sequence" (see text) which defines the sequence of colours expected for O-rich AGB stars surrounded by envelopes with increasing thickness and/or mass loss rates (Bedijn 1987).

The Galactic distribution of the stars in our sample is shown in Fig. 1. The distribution observed clearly suggests that most of them must belong to the disc population. A few sources with bright optical counterparts located at high Galactic latitudes may represent a subset of nearby stars.

In Fig. 2 we show the position of these stars in the IRAS two-colour diagram [12] - [25] vs. [25] - [60]. They fall along the observational "O-rich AGB sequence" (García-Lario 1992), as was expected and with very few exceptions. This sequence agrees very well with the model predictions by Bedijn (1987) and is interpreted as a sequence of increasing thickness of the circumstellar envelopes and/or mass loss rates in O-rich AGB stars.

\section{Observations and data reduction}

The observations were carried out during several observing periods in 1996-97. High-resolution spectra were obtained using the Utrecht Echelle Spectrograph (UES) installed at the Nasmyth focus of the $4.2 \mathrm{~m}$ William Herschel Telescope at the Spanish Observatorio del Roque de los Muchachos (La Palma, Spain) over three different observing runs in August 1996 (run \#1), June 1997 (run \#2) and August 1997 (run \#3) and the CAsegrain Echelle SPECtrograph (CASPEC) of the ESO $3.6 \mathrm{~m}$ telescope at the European Southern Observatory (La Silla, Chile) in February 1997 (run \#4). The full log of the spectroscopic observations is shown in Table 4, including more detailed information on the telescopes, dates of the observations, instrumentation used in each run, spectral dispersion, as well as on the spectral range covered.

We used a TEK $1124 \times 1124$ CCD detector during the first run at the $4.2 \mathrm{~m}$ WHT with UES in August 1996 and a SITe1 $2148 \times 2148 \mathrm{CCD}$ during the second and third runs in June 1997 and August 1997, respectively. Since we were mainly interested in the spectral range between $6000 \AA$ and $8200 \AA$, we used the 31.6 line $/ \mathrm{mm}$ grating in order to provide full coverage of this spectral region in a single spectrum. With a central wavelength around $6700 \AA$ our spectra extended over $\sim 4000 \AA$ during the first run and $\sim 6000 \AA$ during the second and third runs. The spectra were taken with a separation between orders of around 21 pixels (or 7.5"), which is large enough to allow sky subtraction, taking into account that the targets in our sample are nonextended. The resolving power was around 50000 , equivalent to a spectral resolution of $0.13 \AA$ around the Li I line at $6708 \AA$. The selected set-up covered the spectral regions from $5300 \AA$ to $9400 \AA$ in about 47 orders with small gaps in the redder orders for the first run while the 4700-10300 A region was covered in about 61 orders without any gaps for the second and third runs.

CASPEC spectra, taken at the ESO $3.6 \mathrm{~m}$ telescope, covered the wavelength range from $6000 \AA$ to $8200 \AA$. The red crossdisperser (158 line $/ \mathrm{mm}$ ) was used, which gave a resolving power of $\sim 40000$ (equivalent to a spectral resolution of $\sim 0.17 \AA$ around the $\mathrm{Li}$ I line at $6708 \AA$ ) and an adequate interorder separation, using the TEK $1024 \times 1024$ CCD. With this set-up, the selected spectral region is completely covered in 27 orders with small gaps only between the redder ones.

The observational strategy was similar in all runs. Since the stars of the sample are known to be strongly variable, typically 3-4 mag in the $R$-band and sometimes more than 8 mag in the $V$-band, we determined the exposure time according to the brightness of the source at the telescope. The typical exposure times ranged from 10 to $30 \mathrm{~min}$. Two 30-min spectra were taken only for a few very faint stars (and later co-added in order to increase the $S / N$ ratio of the final spectrum). The goal was to achieve an $S / N$ ratio of 50-150 in the region around $6708 \AA$ 


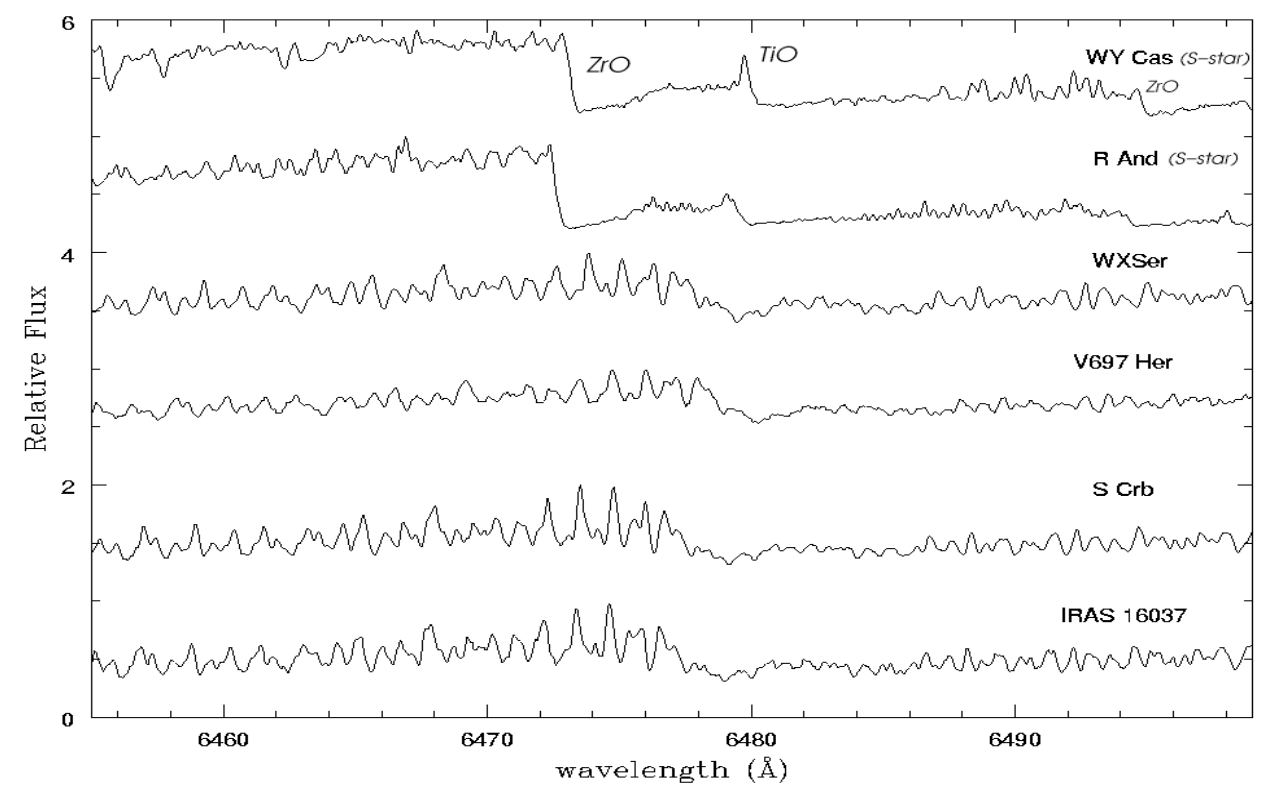

Fig. 3. High-resolution optical spectra of sample stars displaying the lack of the ZrO absorption bands at 6474 and $6495 \AA$ compared with two Galactic S-stars (WY Cas and R And), where these bands are prominent. WX Ser (IRAS 15255+1944) and V697 Her (IRAS 16260+3454) are Li-detected while S CrB (IRAS $15193+3132$ ) and IRAS $16037+4218$ are Li undetected. The absorption band at $\sim 6480 \AA$ corresponds to the $\mathrm{TiO}$ molecule.

(the lithium line) in order to resolve this narrow absorption line, usually veiled at these wavelengths by the characteristic molecular bands which dominate the optical spectrum of these extremely cool stars. Note that, because of the very red colours of the sources observed, the $\mathrm{S} / \mathrm{N}$ ratios achieved for a given star can strongly vary from the blue to the red orders (e.g. 10-20 at $\sim 6000 \AA$ while $>100$ at $\sim 8000 \AA$ ).

At the telescope, all the 102 O-rich stars listed in Table 1 were tried, but useful spectra were obtained only for 57 (56\%). The remaining $45(44 \%)$ sources were either too red to obtain any useful information on the strength of the Li I line at $6708 \AA$ or the optical counterpart was simply not found, in the most extreme cases. Some targets with known, relatively bright optical counterparts in the DSS plates were too faint to be detected on the date of the observations. In contrast, other targets with no optical counterpart in the DSS plates appeared as considerably bright stars. A few Galactic M-supergiants together with some C-, SC-, and S-type AGB stars were also observed for comparison (see list in Table 2). Some of the stars in this latter group are among the most Li-rich stars in our Galaxy. In addition, as we have already mentioned in Sect. 2, a few stars in the initial sample turned out to be carbon stars when observed, and not O-rich as initially suspected (see list in Table 3). The total number of objects observed was 120 .

For wavelength calibration, several Th-Ar lamp exposures were taken every night during the UES runs. In the case of the CASPEC observations, these calibration lamp exposures were taken before or after any science exposure at the position of the target in order to keep control of possible dispersion changes with the telescope position. Note that with UES the system is more stable because the instrumentation is located at the Nasmyth focus of the $4.2 \mathrm{~m}$ WHT telescope. Finally, the corresponding bias and flatfield images were also taken at the beginning (or at the end) of the night.

The two-dimensional frames containing the echelle spectra were reduced to single-order one-dimensional spectra using the standard ECHELLE software package as implemented in
$\mathrm{IRAF}^{4}$. Basically, the data reduction process consists of biaslevel and scattered-light subtraction, the search and trace of apertures using a reference bright star, the construction of a normalized flatfield image to remove pixel-to-pixel sensitivity fluctuations as well as the extraction of the 1D spectra from the $2 \mathrm{D}$ frames. For the wavelength calibration we selected nonsaturated Th-Ar emission lines and third or fourth order polynomials for the fitting. The calibration accuracy reached was always better than $20 \mathrm{~m} \AA$. Finally, we identified the terrestrial features (telluric absorption lines) comparing the target spectra with the spectrum of a hot, rapidly rotating star observed on the same night. It should be noted, however, that the majority of the spectral ranges used in the abundance analysis presented in this paper are not significantly affected by these features.

\section{Results}

\subsection{Overview of the main spectroscopic properties}

In general, all stars show extremely red spectra with the flux level falling dramatically at wavelengths shorter than $6000 \AA$. In addition, the spectra are severely dominated by strong molecular bands mainly due to titanium oxide ( $\mathrm{TiO}$ ), as a consequence of the very low temperature and the O-rich nature of these stars 5 . The bandheads of $\mathrm{TiO}$ at $\sim 6651,6681,6714,7055$, and $7125 \AA$ are clearly present in all spectra. Interestingly, the bandheads of $\mathrm{ZrO}$ at $\sim 6378,6412,6474,6495,6505$, and $6541 \AA$ seem to be absent. These $\mathrm{ZrO}$ bandheads (together with those corresponding to other s-element oxides such as $\mathrm{LaO}$ or $\mathrm{YO}$ ) are very strong in Galactic S-stars (see Fig. 3).

\footnotetext{
${ }^{4}$ Image Reduction and Analysis Facility (IRAF) software is distributed by the National Optical Astronomy Observatories, which is operated by the Association of Universities for Research in Astronomy, Inc., under cooperative agreement with the National Science Foundation.

${ }^{5}$ In contrast, the spectra of the few C-rich AGB stars found in our spectroscopic survey (see list in Table 3) are dominated by $\mathrm{CN}$ and $\mathrm{C}_{2}$ absorption bands.
} 


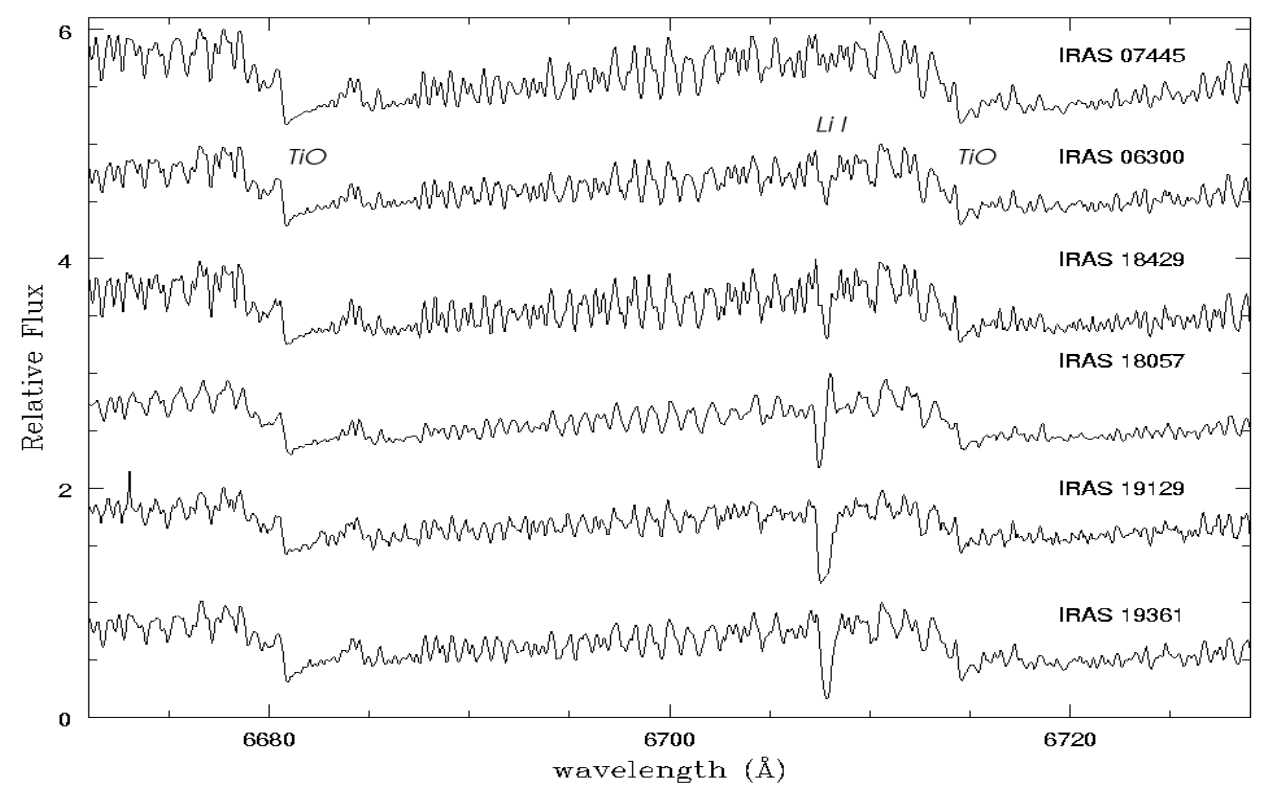

Fig. 4. High-resolution optical spectra of sample stars displaying increasing strength of the Li I line at $6708 \AA$ A. Note the P cygni-type profile of the Li I line in IRAS 18057-2616. The jumps at 6681 and $6714 \AA$ correspond to bandheads of the TiO molecule.

The TiO veiling effect is so intense that it is very difficult to identify individual atomic lines in the spectra of these stars with the exception of the Li I line at $6708 \AA$, the Ca I lines at 6122 and $6573 \AA$, the K I line at $7699 \AA$, the Rb I line at $7800 \AA$, and a few strong Fe I lines. The $\mathrm{K} \mathrm{I}$ and $\mathrm{Rb}$ I resonance lines sometimes show complex profiles, emission over absorption, and blue-shifted components which may have their origin in the expanding circumstellar shell. Actually, the difference between the mean radial velocities of the stellar and circumstellar line components is found to be of the order of the expansion velocity derived from the $\mathrm{OH}$ maser emission. In a few cases the Li I line can also show a similar behaviour (see Fig. 4). Finally, some stars display $\mathrm{H} \alpha$ emission, which in this type of stars is generally interpreted as the consequence of the propagation of shock waves through the outer layers of the stellar atmosphere.

We detected the presence of the $\mathrm{Li}$ I resonance line at $6708 \AA$ in $25 \%$ of the sources in the sample ( 25 stars) with a wide variety of strengths, while in $31 \%$ of them (32 stars) we did not find any signature of this line. The remaining $44 \%$ (45 stars) were either too red or the optical counterpart was simply not found at the moment of the observations. Sample spectra of stars showing increasing strength of the Li I line at $6708 \AA$ are presented in Fig. 4.

The observed sample has been divided into two different groups on the basis of the detection or non-detection of the lithium line in Tables 5 and 6 , respectively. A third group is formed by the stars that were too red (or which did not show any optical counterpart at the telescope), and these are listed in Table 7. The IRAS name, the run in which the stellar field was observed, the $\mathrm{OH}$ expansion velocity, and the pulsational period (if available) are given for every source in these tables. The same information is also presented for the comparison stars in Table 8 and for the sample of peculiar C-rich AGB stars in Table 9 (in this latter case the expansion velocities are derived from CO data).

The detection of strong circumstellar $\mathrm{Li} \mathrm{I}$ and $\mathrm{Rb}$ I lines in S Per (IRAS 02192+5821), sometimes classified in the literature as an M-type Galactic supergiant, is remarkable. M-type supergiant stars are not expected to show a strong Li I line (e.g.
Luck \& Lambert 1982) or any s-process element enhancement (e.g. Smith et al. 1995). According to our data, S Per looks like a possible genuine O-rich AGB star. In addition, we find a cool effective temperature of $\sim 3000 \mathrm{~K}$ which is quite different from Levesque et al. (2005) and further investigation is needed. Similarly, PZ Cas (IRAS 23416+6130), another source usually classified as an M-type supergiant (e.g. Levesque et al. 2005), is for the first time clearly identified by us as a possible S-,SC-type AGB star. PZ Cas seems to be slightly enriched in $\mathrm{Zr}$ and displays some strong $\mathrm{Ba}$ atomic lines but it is not enriched in $\mathrm{Li}$, as expected if $\mathrm{PZ}$ Cas is a low-mass S-,SC-type AGB star. Consistent with our possible AGB identification for the latter two sources, they show variability amplitudes of more than $3 \mathrm{mag}$ in the $V$-band and are the only two stars with periods well beyond 500 days in Table 8. A more detailed analysis of these two stars is beyond the scope of this paper but it would be needed to reach a definitive conclusion. Also interesting is the new detection of a strong lithium line in the peculiar mixed chemistry (C-rich and O-rich) star IRAS 09425-6040, which is studied in a separate paper (García-Hernández et al. 2006a). Lithium was also detected in the C-rich AGB stars IRAS 04130+3918, IRAS 20072+3116 (V1969 Cyg), and IRAS 23320+4316 (LP And), for which a detailed analysis will be presented elsewhere. Finally, the S-star IRAS 10436-3459 (Z Ant), observed for the first time with highresolution spectroscopy, shows, as expected, intense molecular bands of s-element oxides (like $\mathrm{ZrO}$ and $\mathrm{LaO}$ ) but no lithium. This star will also be analysed in detail in a separate publication.

\subsection{Modelling strategy}

Atmospheres of cool pulsating AGB stars present a major challenge for realistic, self-consistent modelling. At present, two types of self-consistent models exist. Classical hydrostatic model atmospheres include a sophisticated treatment of microphysical processes and radiative transfer but neglect the effects of dynamics due to pulsation and winds (e.g. Plez et al. 1992). Time-dependent dynamical models for stellar winds (Winters et al. 2000; Höfner et al. 2003, and references therein) include 
pulsation, dust formation but a more crude description of radiative transfer. Propagating shock waves caused by stellar pulsation modify the structure of the atmosphere on local and global scales, which give rise to strong deviations from a hydrostatic stratification. The radiative field is dominated by the effect of molecular opacities or even by the dust grains forming in the cool outer layers of these atmospheres. Chemistry and dust formation may be severely out of equilibrium.

Hydrostatic model atmospheres based on the MARCS code (Plez et al. 1992; Gustafsson et al. 2003) reproduce well the observed spectra of C-rich AGB stars (e.g. Loidl et al. 2001) and O-rich AGB stars (e.g. Alvarez et al. 2000) in the optical domain and were thus adopted for the analysis. Indeed, the optical spectra of massive O-rich AGB stars in the MCs have succesfully been modelled using this code (e.g. Plez et al. 1993). A comparison of these models with high-resolution observational data in the optical for massive O-rich AGB stars in our Galaxy is unfortunately still lacking.

Our analysis combines state-of-the-art line-blanketed model atmospheres and synthetic spectroscopy with extensive line lists. For this we need to estimate first the range of values of the stellar parameters that can reasonably be adopted for the stars in our sample: effective temperature $T_{\text {eff }}$, surface gravity $\log g$, mass $M$, metallicity $Z=[\mathrm{Fe} / \mathrm{H}]$, microturbulent velocity $\xi$, and $\mathrm{C} / \mathrm{O}$ ratio.

Spherically symmetric, LTE, hydrostatic model atmospheres were calculated using the MARCS code for cool stars (Gustafsson et al. 2003). The models are designed with the notation $\left(T_{\text {eff }}, \log g, M, Z, \xi, \mathrm{C} / \mathrm{O}\right)$, where $T_{\text {eff }}$ is the effective temperature of the star in $\mathrm{K}, g$ is the surface gravity in $\mathrm{cm} \mathrm{s}^{-2}, M$ is the stellar mass in $M_{\odot}, Z$ is the metallicity, $\xi$ is the microturbulent velocity in $\mathrm{km} \mathrm{s}^{-1}$, and $\mathrm{C} / \mathrm{O}$ is the ratio between the $\mathrm{C}$ and $\mathrm{O}$ abundances.

Synthetic spectra were generated with the TURBOSPECTRUM package (Alvarez \& Plez 1998) which shares much of its input data and routines with MARCS. Solar abundances of Grevesse \& Sauval (1998) were always adopted, except for the iron abundance, which was taken as $\log \varepsilon(\mathrm{Fe})=7.50$. Most opacity sources for cool M-type stars were included and taken from the literature. In addition, updated line lists were produced by us for $\mathrm{TiO}$ (Plez 1998), ZrO (Plez et al. 2003), and VO (Alvarez \& Plez 1998).

For atomic lines, the primary source of information was the VALD-2 database (Kupka et al. 1999). The NIST ${ }^{6}$ atomic spectra database was also consulted for comparison. Where possible, $g f$ values were checked by fitting the solar spectrum. VALD-2 $g f$ values taken from Kurucz $(1993,1994)$ were often erroneous and did not fit the atomic lines of the solar spectrum. The identification of features was made using the solar atlases by Moore et al. (1966) and Wallace et al. (1993, 1998) and the observed solar spectrum of Neckel (1999). The TURBOSPECTRUM program was run using the abundances from Grevesse \& Sauval (1998) together with the solar model atmosphere by Holweger \& Müller (1974) with parameters $T_{\text {eff }}=5780 \mathrm{~K}, \log g=4.44$, and a variable microturbulence as a function of the optical depth.

The whole machinery was tested on the high-resolution optical spectrum $(R \sim 150000)$ of the K2 IIIp giant Arcturus ( $\alpha$ Boo) from Hinkle et al. (2000). A MARCS model atmosphere was used with the fundamental parameters determined by Decin et al. (2003a): $T_{\text {eff }}=4300 \mathrm{~K}, \log g=1.50, Z=-0.5, M=0.75 M_{\odot}$, $\xi=1.7 \mathrm{~km} \mathrm{~s}^{-1}, \mathrm{C} / \mathrm{N} / \mathrm{O}=7.96 / 7.55 / 8.67$, and ${ }^{12} \mathrm{C} /{ }^{13} \mathrm{C}=7$. These parameters are in excellent agreement with other determinations

6 National Institute of Standards and Technology (NIST); http://physics.nist.gov/cgi-bin/AtData/lines_form. reported in the literature. Some Kurucz $g f$ values of metallic lines of Fe I, Ni I, Co I, CrI, Ti I, ZrI, and Nd II, which were too weak in the solar spectrum, were adjusted so as to yield a good fit to the Arcturus spectrum in several regions (especially in the 7400-7600 $\AA$ and 8100-8150 $\AA$ regions); otherwise we used the $g f$ values from the VALD-2 database. This exercise was very useful in confirming the lack of s-process atomic lines in the 7400-7600 and 8100-8150 ̊ spectral windows in the Galactic O-rich AGB stars in our sample, as we shall see later.

\subsection{Spectral regions of interest}

For the abundance analysis we concentrated our attention on the following spectral regions.

\subsubsection{Lithium}

We used the Li I resonance line at $\sim 6708 \AA$ to derive the lithium abundances, which can be used as a signature of HBB. The selected spectral region ranges from 6670 to $6730 \AA$ and covers the TiO molecular bandheads at $\sim 6681$ and $6714 \AA$, which are sensitive to variations in the effective temperature. In addition, the spectral regions around the subordinate Li I lines at $\sim 6103$ and $8126 \AA$ were also inspected. However, we decided to drop them from the analysis because the latter lines are much weaker. Moreover, the S/N ratio is usually very low around $6103 \AA$ in our sources and the contamination by telluric lines at $8126 \AA$ is very strong.

\subsubsection{Rubidium and potassium}

We also synthesized the spectral regions around the resonance lines of $\mathrm{Rb}$ I at $\sim 7800 \AA$ and K I at $7699 \AA$, with the intention of using the elemental abundances derived from these lines as neutron density and metallicity indicators, respectively. In addition, these spectral regions were also used to check that the model parameters adopted to reproduce the lithium region also provided a reasonably good fit at other wavelengths. Two intervals covering $\sim 60 \AA$ in the regions $7775-7835 \AA$ and 7670-7730 ̊ were selected for this purpose. Unfortunately, the frequent detection of circumstellar components to these lines prevented the accurate determination of the $\mathrm{K}$ I elemental abundances, and they will not be discussed here. To a lesser extent the problem also affects the $\mathrm{Rb}$ I line. A detailed analysis of the $\mathrm{Rb}$ abundances will be presented in a forthcoming paper (García-Hernández et al. 2006b).

\subsection{3. s-process elements}

The study of the s-process elements was carried out through the analysis of several $\mathrm{ZrO}$ absorption bands in the region from 6455 to $6499 \AA$. In particular, we used the ZrO bandhead around $\sim 6474 \AA$ to determine severe upper limits to the $\mathrm{Zr}$ elemental abundance, taken as representative of the overall s-process element enrichment. Surprisingly, this band was not detected in any star in our sample, neither did we find any signature from atomic $\mathrm{Zr}$ or from atomic lines corresponding to any other abundant s-process element in the 7400-7600 $\AA$ and 8100-8150 $\AA$ spectral windows, where many atomic lines of $\mathrm{Zr}$ I, Nd II, Ba II might potentially have been found, as we show later. 


\subsection{Derivation of the stellar parameters}

In order to analyze how the variations in stellar parameters influence the output synthetic spectra, we constructed a grid of MARCS model atmospheres and generated the associated synthetic spectra with the following specifications: i) the stellar mass was in all cases taken to be $2 M_{\odot}$; ii) $T_{\text {eff }}$ values ranging from 2500 to $3800 \mathrm{~K}$ in steps of $100 \mathrm{~K}$; iii) $\mathrm{C} / \mathrm{O}$ ratio values of $0.5,0.7,0.8$, and 0.9 ; iv) $\log g$ between -0.5 and $1.6 \mathrm{dex}$ in steps of $0.3 \mathrm{dex}$; v) microturbulent velocity $\xi$ between 1 and $6 \mathrm{~km} \mathrm{~s}^{-1}$ in steps of $0.5 \mathrm{~km} \mathrm{~s}^{-1}$; vi) metallicity $Z$ between 0.0 and $-0.3 \mathrm{dex}$; vii) $\log \varepsilon(\mathrm{Zr})^{7}$ from 1.6 to $3.6 \mathrm{dex}$ in steps of 0.25 dex; viii) $\mathrm{CNO}$ abundances shifted $\pm 1.0 \mathrm{dex}$ (in steps of 0.5 dex) from the solar values of Grevese \& Sauval (1998); and ix) ${ }^{12} \mathrm{C} /{ }^{13} \mathrm{C}$ ratios of 10 (as expected for HBB stars) and 90 (the solar value). Finally, the synthetic spectra were convolved with a Gaussian profile (with a certain FWHM typically between 200 and $600 \mathrm{~m} \AA$ ) to account for macroturbulence as well as instrumental profile effects.

This, however, results in an extremely large grid of synthetic spectra containing thousands of possible combinations of the above parameters! In order to reduce the number of spectra to be considered in our analysis, further constraints were imposed by studying the sensitivity of our spectral synthesis to changes in these parameters.

\subsubsection{Stellar mass}

As we have already mentioned, the stellar mass was in all cases considered to be constant and equal to $2 M_{\odot}$. This is because the temperature and pressure structure of the model atmosphere is practically identical for a $1 M_{\odot}$ and a $10 M_{\odot}$ model atmosphere (see Fig. 1 in Plez 1990). Increasing the mass will just have a marginal effect on the output synthetic spectrum through a decrease, for a given gravity, of the atmospheric extension.

\subsubsection{Effective temperature}

The synthetic spectra are, in contrast, particularly sensitive to $T_{\text {eff }}$, which determines the overall strength of the molecular absorption over the continuum (mainly, from TiO molecules but also from $\mathrm{VO}$, and from $\mathrm{ZrO}$ if the $\mathrm{Zr}$ elemental abundance is increased above a certain limit). Thus, we decided to keep the whole range of values initially considered in our grid, i.e. $T_{\text {eff }}$ from 2500 to $3800 \mathrm{~K}$ in steps of $100 \mathrm{~K}$. Actually, the depth of the molecular bands increases considerably with decreasing temperature. The effective temperature has also a large impact on the strength of the Li I line at $6708 \AA$ in the synthetic spectrum. A decrease of $T_{\text {eff }}$ has the effect of increasing the TiO veiling, as well as the strength of the Li I line, as a consequence of the changes in the $\mathrm{Li} \mathrm{I} / \mathrm{Li}$ II population equilibrium.

\subsubsection{C/O ratio}

The C/O ratio basically determines the prevalence of O-rich molecules $\mathrm{C} / \mathrm{O}<1$ ) against $\mathrm{C}$-rich molecules like $\mathrm{CN}, \mathrm{C}_{2}$, etc. In the case of our O-rich AGB stars, the precise value of the $\mathrm{C} / \mathrm{O}$ ratio adopted, always $<1$, affects mainly the strength of the $\mathrm{TiO}$ veiling and of the $\mathrm{Li}$ I line in the synthetic spectrum. An increase of the $\mathrm{C} / \mathrm{O}$ ratio will decrease the $\mathrm{TiO}$ veiling and increase the $\mathrm{Li}$ I line strength relative to the adjacent continuum in a very

7 The zirconium abundance is given in the usual scale $12+\log N(\mathrm{Zr})$. On this scale the solar zirconium abundance is $2.6 \mathrm{dex}$. similar way as a decrease in $T_{\text {eff. }}$ This is a good example of how a set of parameters providing a good match to the observations for a given spectral region is not necessarily unique. A good fit may be obtained for another combination of parameters that can correspond to quite different Li abundances. Fortunately, one of these two sets of parameters usually does not provide acceptable fits for other spectral regions. In particular, the presence of detectable atomic lines in the synthetic spectrum is very sensitive to variations in the $\mathrm{C} / \mathrm{O}$ ratio: an increase in the $\mathrm{C} / \mathrm{O}$ ratio will lower the $\mathrm{TiO}$ veiling, making the detection of atomic lines easier. According to the models, the immediate effect should be the detection of much stronger atomic lines of K I, Fe I, Zr I, Nd II, etc. Since we do not see any such effect in our spectra, we conclude that $\mathrm{C} / \mathrm{O}$ must always be $\leq 0.75$ in our stars. Abundances derived from models with $\mathrm{C} / \mathrm{O}$ between 0.15 and 0.75 show actually little differences (Plez et al. 1993). Given that all stars in our sample are clearly O-rich and taking into account the above considerations, we decided to use $\mathrm{C} / \mathrm{O}=0.5$ in all cases, as a constant value. This selection is in agreement with other more detailed determinations made in massive O-rich AGB stars in the MCs (e.g. Plez et al. 1993; Smith et al. 1995) and in a few low-mass M-type stars studied in our Galaxy (Smith \& Lambert $1985,1990 \mathrm{~b})^{8}$.

\subsubsection{Surface gravity}

The surface gravity also affects the appearance of the output synthetic spectra but its effect is small compared to that of the effective temperature. The influence of a change in the value adopted for the surface gravity within the range of values (log $g$ between -0.5 and 0.5 dex) under consideration is not as severe as a temperature change. The molecular absorption becomes slightly weaker at higher surface gravities. For example, an increase in the surface gravity of $0.5 \mathrm{dex}$ has approximately the same impact as an increase of $100 \mathrm{~K}$ in effective temperature. However, for a fixed temperature we found that the lowest gravities in general fit better both the $\mathrm{TiO}$ band strength and the pseudocontinuum around the $6708 \AA \mathrm{Li}$ I line. As the appearance of the spectra is not so dependent on the surface gravity, and considering that its value must be low in these mass-losing stars, a constant surface gravity of $\log g=-0.5$ was adopted for all the stars in the sample. This selection seems to be also appropriate if it is compared with the values adopted by Plez et al. (1993) of $-0.33 \leq \log g \leq 0.0$ for O-rich AGB stars in the MCs with effective temperatures between 3300 and $3650 \mathrm{~K}$.

\subsubsection{Microturbulent velocity}

The microturbulent velocity is usually derived by demanding no correlation between the abundance of iron derived from individual lines and their reduced equivalent widths. Previous studies of O-rich AGB stars found microturbulent velocities between 2 and $4 \mathrm{~km} \mathrm{~s}^{-1}$ (Plez et al. 1993; Smith \& Lambert 1985, 1989; Smith et al. 1995; Vanture \& Wallerstein 2002). Unfortunately, we cannot estimate the microturbulent velocity in the stars of our sample due to the lack of useful Fe I atomic lines (the small number of detectable Fe I lines does not cover a wide range of

8 This is the only other chemical analysis previously carried out in Galactic O-rich AGB M-type stars (only six stars!) before the work presented here. However, these were low-mass stars $\left(M \lesssim 1.5-2 M_{\odot}\right)$ and their study was focussed on the determination of CNO abundances through near-IR spectra and the s-process enrichment, but they did not study the lithium enrichment in particular. 
equivalent widths). Thus, we will assume in the following a microturbulent velocity $\xi=3 \mathrm{~km} \mathrm{~s}^{-1}$, which is a typical value generally adopted for AGB stars (e.g. Aringer et al. 2002). Higher values of $\xi$ would strenghten all atomic lines in the synthetic spectra and we do not see this effect in our data.

\subsubsection{Metallicity}

The metallicity of the Galactic O-rich AGB stars in our sample is assumed to be solar, as expected for stars belonging to the disc population of our Galaxy. A lower metallicity is unlikely since all the stars are expected to be at least of intermediate mass $\left(M \gtrsim 2-3 M_{\odot}\right)$, if not more massive. This assumption is also in good agreement with the typical metallicities derived for other AGB stars in our Galaxy (e.g. Abia \& Wallerstein 1998; Vanture \& Wallerstein 2002). The Ca I lines at $\sim 6122 \AA$ and $6573 \AA$ generally used for this determination in other studies are unfortunately not sensitive enough to metallicity nor to surface gravity at the very low temperature of our sample stars (see Fig. 7 of Cenarro et al. 2002). This was checked by running different test models and spectral syntheses in the 6100-6160 ̊ and 6535-6585 $\AA$ regions where these lines fall. A decrease in the metallicity implies less availability of metals like $\mathrm{Ti}$, and thus, lowers the $\mathrm{TiO}$ veiling, making the detection of atomic lines easier. Actually, this may be the main reason why they are easily detected in O-rich AGB stars at the metallicity of the MCs (e.g. $Z=-0.5$ in the SMC, Plez et al. 1993), while we do not detect them in our spectra.

\subsubsection{Zirconium}

The synthetic spectra are also very sensitive to changes in the zirconium elemental abundance, especially in those wavelength regions where $\mathrm{ZrO}$ molecular bands are present. For a set of fixed stellar parameters, an increase in the zirconium abundance leads to stronger bands. Since the zirconium abundance was a priori unknown in our stars, we decided to use a large set of zirconium abundances with $\log \varepsilon(\mathrm{Zr})$ between 1.6 and $3.6 \mathrm{dex}$ in steps of $0.25 \mathrm{dex}$. The effect of a variation in the zirconium elemental abundance on the $\mathrm{ZrO}$ bands is in general much stronger than any other effect associated with variations in the $T_{\text {eff }}$ or $\mathrm{C} / \mathrm{O}$ ratio. In contrast, even at relatively high zirconium abundance, the corresponding $\mathrm{Zr}$ atomic lines are more difficult to detect at low $T_{\text {eff }}$ or low $\mathrm{C} / \mathrm{O}$ ratio because the $\mathrm{TiO}$ and $\mathrm{ZrO}$ lines become stronger and more numerous under these conditions compared to the $\mathrm{Zr}$ atomic lines.

\subsubsection{CNO abundances and ${ }^{12} \mathrm{C} /{ }^{13} \mathrm{C}$ ratio}

In the synthetic spectra, relative variations in the carbon, nitrogen, and oxygen abundances and of the ${ }^{12} \mathrm{C} /{ }^{13} \mathrm{C}$ ratio are completely negligible with respect to other stellar fundamental parameters in the spectral regions under analysis. Thus, we decided to fix them and adopt their solar values in the following analysis.

\subsection{Abundance determination}

\subsubsection{Overall strategy}

After imposing these further constraints, the grid of MARCS model spectra to consider is composed of "only" a few hundred spectra, with effective temperatures in the range $T_{\text {eff }}=$ $2500-3800 \mathrm{~K}$ in steps of $100 \mathrm{~K}, \log \varepsilon(\mathrm{Zr})$ between 1.6 and 3.6 dex in steps of $0.25 \mathrm{dex}$, and a variable value of the FWHM in the range 200-600 $\mathrm{m} \AA$ in steps of $50 \mathrm{~m} \AA$, keeping all the other stellar parameters fixed: $\log g=-0.5, M=2 M_{\odot}$, solar metallicity $Z=[\mathrm{Fe} / \mathrm{H}]=0.0, \xi=3 \mathrm{~km} \mathrm{~s}^{-1}, \mathrm{C} / \mathrm{O}=0.5$, solar CNO abundances, and solar ${ }^{12} \mathrm{C} /{ }^{13} \mathrm{C}$ ratios.

In order to find the synthetic spectrum which better fits the observed spectrum of a given star a modified version of the standard $\chi^{2}$ test was used. When fitting observed data $Y_{\text {obsi }}$ to model data $Y_{\text {synthi }}$, the quality of the fit can be quantified by the $\chi^{2}$ test. The best fit corresponds to that leading to the minimum value of $\chi^{2}$. This test is used here in a modified way and is mathematically expressed as:

$\chi^{2}=\sum_{i=1}^{N} \frac{\left[Y_{\text {obsi }}-Y_{\text {synthi }}\left(x_{1} \ldots x_{M}\right)\right]^{2}}{Y_{\text {obsi }}}$

with $N$ the number of data points and $M$ the number of free parameters.

The observed spectra, once fully reduced, were shifted to rest-wavelength using the mean radial velocity shift derived from the Li I and $\mathrm{Ca}$ I lines. In addition, they were re-binned to the same resolution as the synthetic ones $(0.06 \AA /$ pix $)$ and normalized in order to make the comparison easier. The observed spectra were then compared to the synthetic ones in intervals of $\sim 60 \AA$ which allowed the analysis of their overall characteristics as well as of the relative strength of the TiO molecular bands.

The fitting procedure makes a special emphasis on the goodness of the fit in the lithium region (6670-6730 $\AA$ ). In this spectral range the goal was to fit the relative strength of the TiO bandheads at $\sim 6681$ and $6714 \AA$, which are very sensitive to the effective temperature, together with the pseudo-continuum around the Li I line. We first determined by $\chi^{2}$ minimization which of the spectra from our grid of models provided the best fit, mainly fixing $T_{\text {eff }}$. The best fits resulting from the $\chi^{2}$ minimization were also judged by eye in order to test the method. The lithium content was then estimated by changing the lithium abundance. This procedure was repeated on each star of the sample for which an acceptable $S / N$ ratio was achieved around the Li I $6708 \AA$ line. Unfortunately, we could not analyse a few AGB stars with a low signal-to-noise ratio in their spectra at $6708 \AA$ (see Table 7). IRAS 18025-2113, IRAS 03507+1115, and IRAS 18304-0728 showed unusual spectra, very different from the rest of the stars in our sample and a good fit from our grid of MARCS model spectra was not possible. The spectra of these stars show unusual $\mathrm{TiO}$ band strengths which could only be reproduced with a low $T_{\text {eff }}$ but the rest of the spectrum (e.g. the local continuum level) seems to be hotter. In addition, the atomic lines are broader (IRAS 18025-2113 and IRAS 18025-2113) or narrower (IRAS 03507+1115) than the FWHM needed to describe the TiO bandheads and lines. Their complicated spectra look like a combination of two temperatures and FWHMs suggesting that they could probably be doublelined spectroscopic binaries or strongly affected by shock waves propagating in their atmosphere.

The best model fit in the Li I spectral region (6670-6730 ̊) is also usually found to fit the wavelength regions around the $\mathrm{ZrO}$ bandhead and the $\mathrm{K} \mathrm{I}$ and $\mathrm{Rb}$ I resonance lines reasonably well. In general, the overall shape of the spectrum (including the TiO bandheads) is very well reproduced. As an example, the fits made in different spectral regions for the star IRAS 11081-4203 are presented in Figs. 5 to 9. The effective temperatures of the best-fitting model spectra together with the lithium abundances 


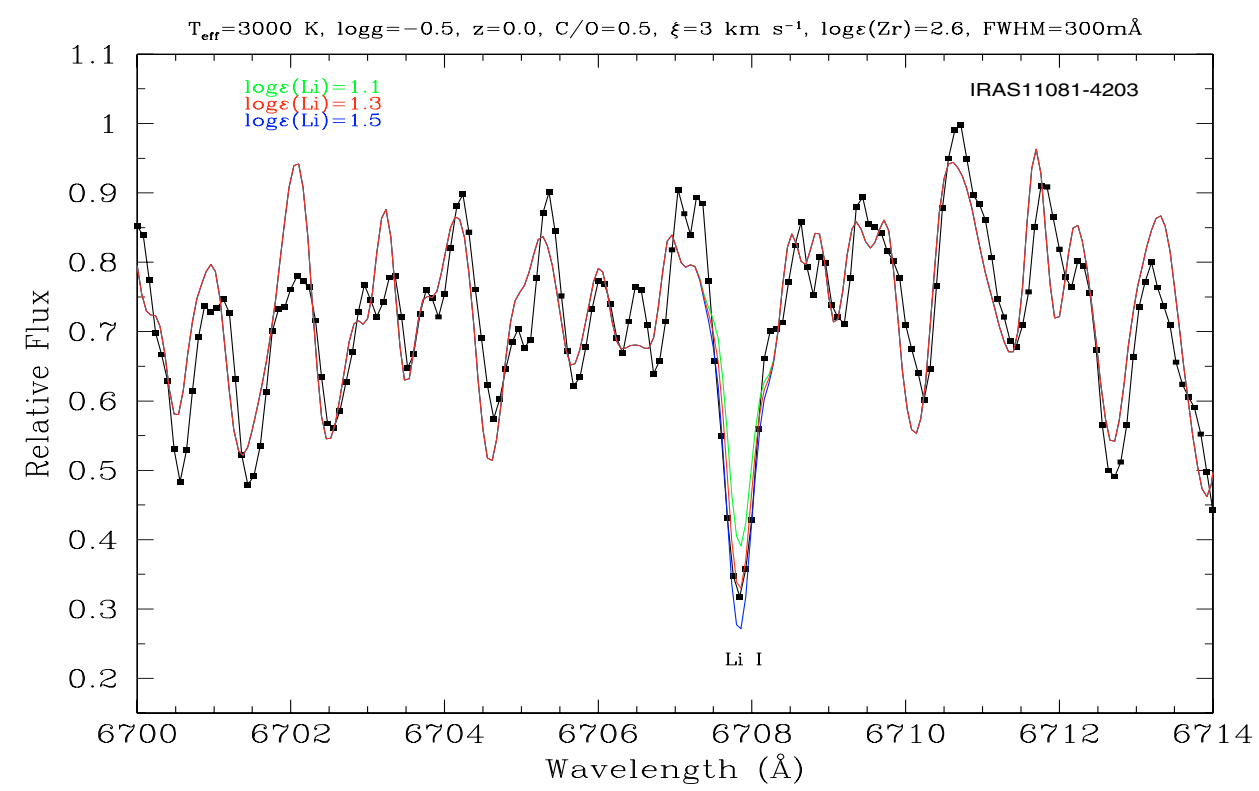

Fig. 5. Best model fit and observed spectrum around the Li I line (6708 $⿱$ ) for the star IRAS 11081-4203. The Li abundance derived from this spectrum was $\log \varepsilon(\mathrm{Li})=1.3 \mathrm{dex}$. The synthetic spectra obtained for $\mathrm{Li}$ abundances shifted +0.2 dex and -0.2 dex from the adopted value are also shown. The parameters of the best model atmosphere fit are indicated in the top label.

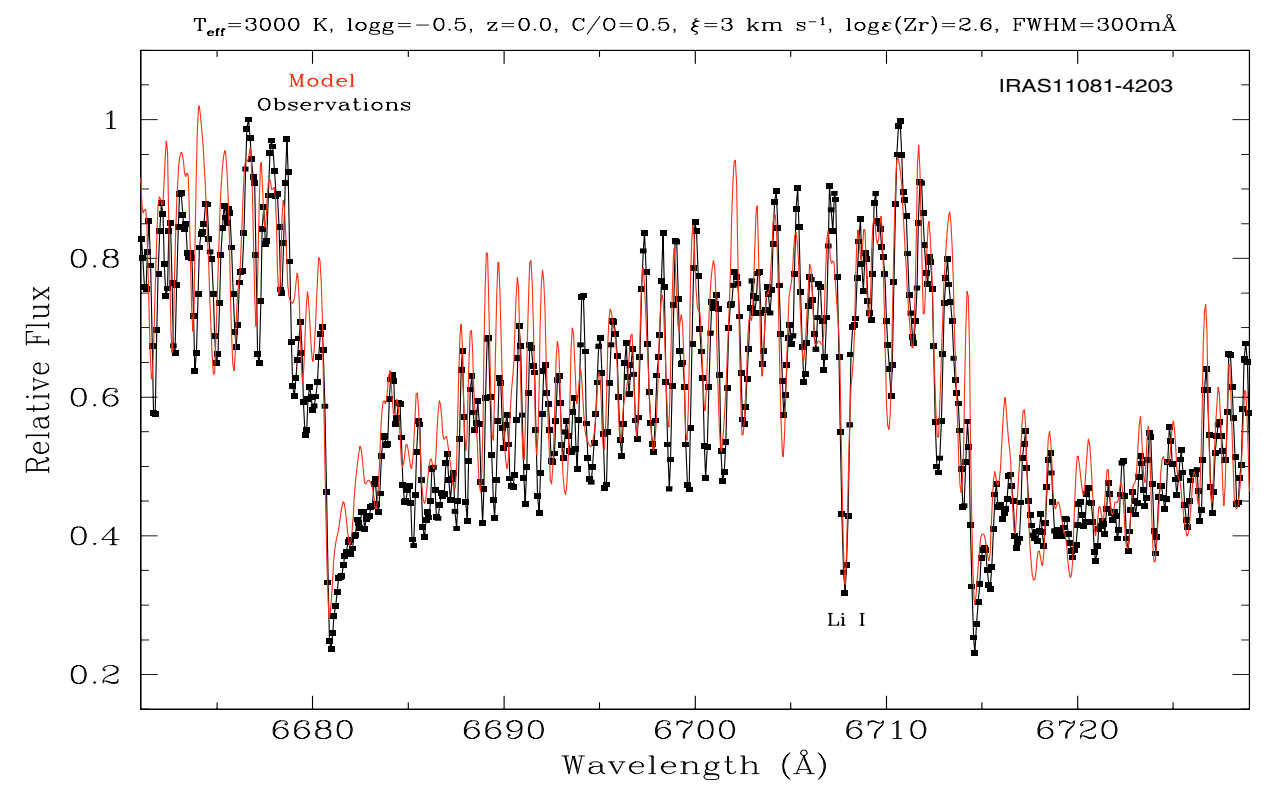

Fig. 6. Best model fit and observed spectrum in the region 6670-6730 ̊ for the star IRAS 11081-4203. The $T_{\text {eff }}$ derived from this spectrum was $3000 \mathrm{~K}$. The parameters of the best model atmosphere fit are indicated in the top label.

(or upper limits) derived following the above procedure are listed in Table 10, where we have separated the Li detected stars from the $\mathrm{Li}$ undetected ones.

For the measurement of the zirconium abundance we concentrated our attention on the synthesis of the 6455-6499 spectral region. In this case, the goal was to obtain the best fit around the $\mathrm{ZrO}$ molecular bands at $6474 \AA$ and $6495 \AA$. The non-detection of these features in any of the stars under analysis imposes severe upper limits to the zirconium abundance. To be sure that there was no other way to interpret our data, we also checked the presence of atomic zirconium in the 7400-7600 and 8100-8150 ̊ spectral regions, where strong Zr I atomic lines should have been detected in that case. Unfortunately, the local continuum in these latter wavelength regions is not well reproduced in the O-rich AGB stars in our sample, in contrast with the perfect agreement obtained in some of the comparison stars observed, such as the S-star IRAS 10436-3454, for which a perfect fit is obtained both for the $\mathrm{ZrO}$ bandheads at 6455-6499 $\AA$ (see Fig. 12) as well as for the Zr I atomic lines in the 7400-7600 and 8100-8150 $\AA$ spectral regions (not shown). Sample spectra of the region around $7565 \AA$ are shown in Fig. 10, where the position of some atomic lines of $\mathrm{Zr}$ I, Nd II, and $\mathrm{Fe} \mathrm{I}$ are indicated. As we can see, the atomic lines corresponding to s-process elements (e.g. $\mathrm{Zr}, \mathrm{Nd}$, La, etc.) were not detected in any of the sample stars observed. In contrast, these s-element atomic lines appear very strong in the Galactic S-star IRAS 10436-3454. Note, however, that the stars in our sample are the coolest O-rich AGB stars ever studied (with effective 


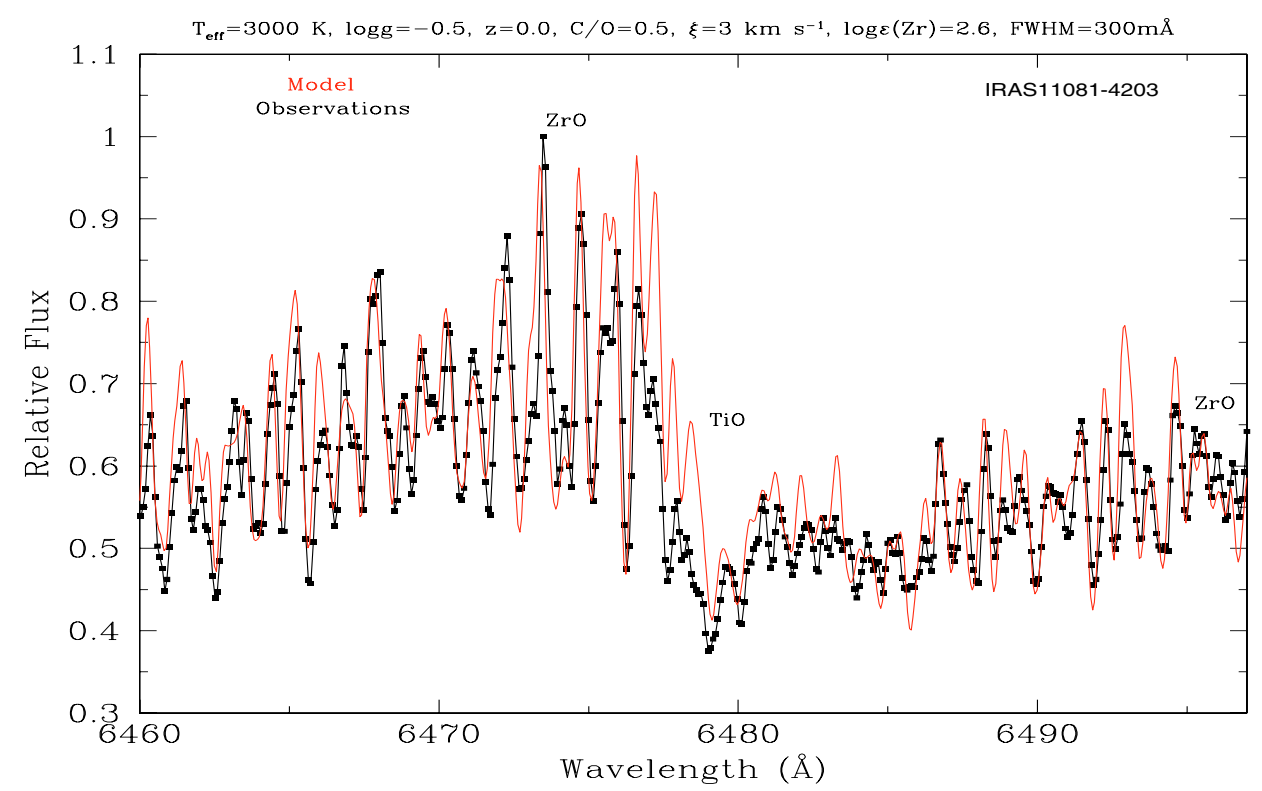

Fig. 7. Best model fit and observed spectrum in the region 6460-6499 $\AA$ for the star IRAS 11081-4203. The Zr abundance derived from this spectrum was $\log \varepsilon(\mathrm{Zr})=2.6 \mathrm{dex}$, which corresponds to the solar value. The parameters of the best model atmosphere fit are indicated in the top label. Note the non-detection of the characteristic strong $\mathrm{ZrO}$ bandheads typical of Galactic S-stars.

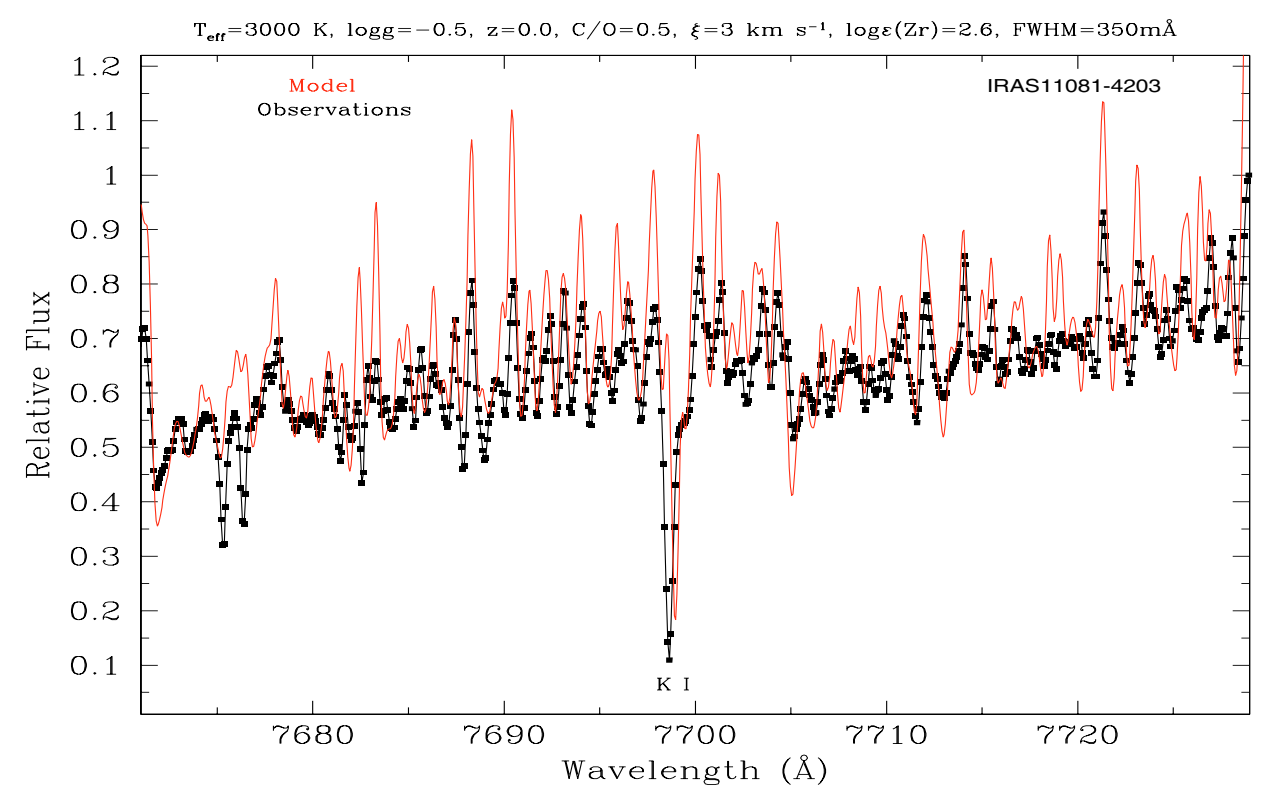

Fig. 8. Best model fit and observed spectrum in the region 7670-7730 A (around the K I line at $7699 \AA$ ) for the star IRAS 11081-4203. The synthetic spectrum obtained for solar $\mathrm{K}$ abundance $(\log \varepsilon(K)=5.12 \mathrm{dex})$ is shown. The parameters of the best model atmosphere fit are indicated in the top label. Note the radial velocity shift, which is interpreted as a result of the partially circumstellar origin of the K I line. The narrow features seen at $\sim 7675-77 \AA$, which are not adjusted by the model, are telluric lines.

temperatures between $2700 \mathrm{~K}$ and $3300 \mathrm{~K}$; see Table 10). We suspect that the strong discrepancies observed between model and observations may be due to the non-inclusion of other $\mathrm{O}$ rich molecules, such as $\mathrm{H}_{2} \mathrm{O}$, in our line lists. The effect of $\mathrm{H}_{2} \mathrm{O}$ may be ignored in $\mathrm{S}$-stars, where the $\mathrm{C} / \mathrm{O}$ ratio is close to unity, but it might become dominant in our O-rich stars at these very low temperatures, preferentially at the longer wavelengths (e.g. Allard et al. 2000; Decin et al. 2003a,b). In addition, lanthanum oxide also has a strong absorption band around $7500 \AA$ and it could have been a good candidate for detection in the spectral range here considered. As for $\mathrm{ZrO}$, the non-detection of the strong $\mathrm{LaO}$ molecular bandhead, usually very strong in $\mathrm{S}$-stars at these wavelengths, is another indication of the lack of s-process elements in our sample stars.

\subsubsection{Lithium and s-element abundances}

The derived $\mathrm{Li}$ abundances are displayed in Table 10, where we can see that almost all Li detected stars show enhanced Li abundances $\log \varepsilon(\mathrm{Li}) \geq 1$, i.e. larger than solar, but smaller than those found in the so-called "super Li-rich" AGB stars (with $\log \varepsilon(\mathrm{Li})>3-4$; e.g. Abia et al. 1991) in our Galaxy. A very similar range of $\mathrm{Li}$ overabundances was found in the massive O-rich AGB stars studied in the MCs (Plez et al. 1993; 


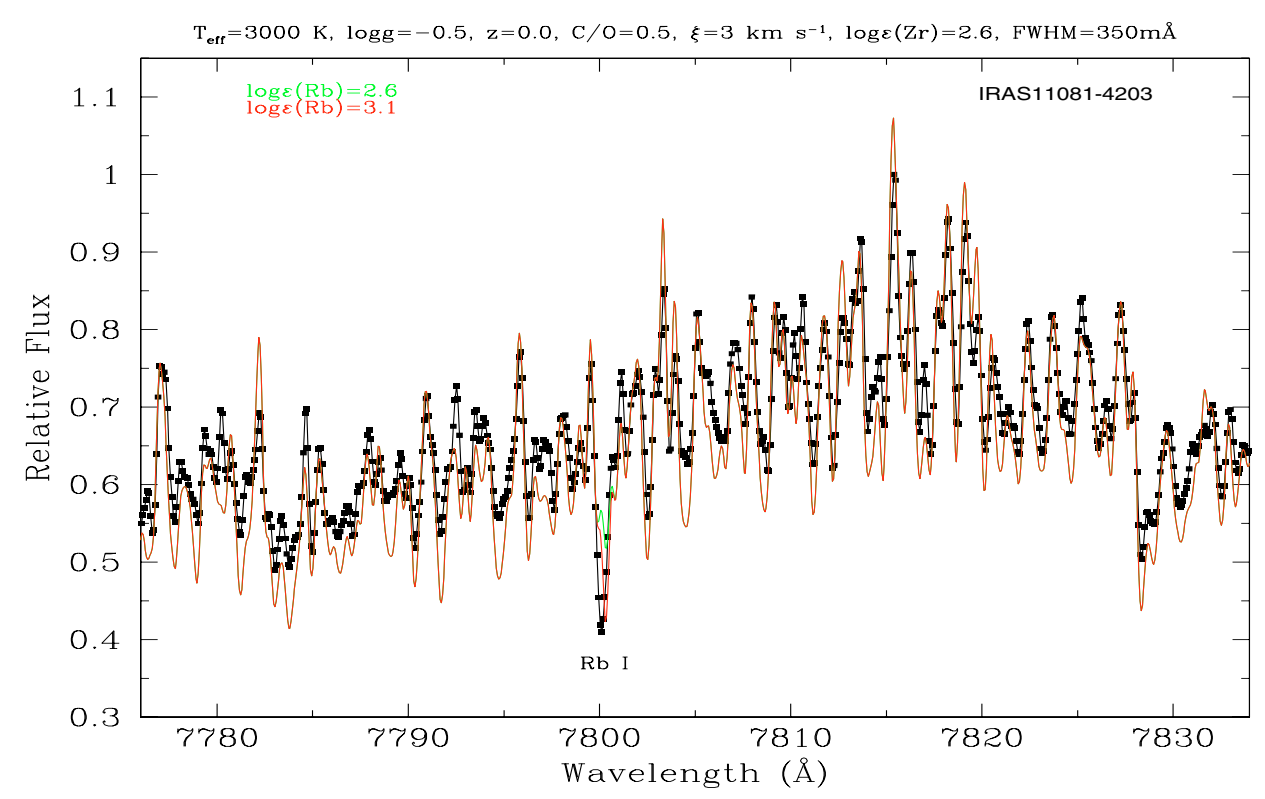

Fig. 9. Best model fit and observed spectrum in the region $7775-7835 \AA$ (around the Rb I line at $7800 \AA$ ) for the star IRAS $11081-4203$. The synthetic spectra obtained for solar Rb abundance $(\log \varepsilon(\mathrm{Rb})=2.6$ dex $)$ and shifted $+0.5 \operatorname{dex}(\log \varepsilon(\mathrm{Rb})=3.1$ dex $)$ are shown. The parameters of the best model atmosphere fit are indicated in the top label. Again, the radial velocity shift observed suggests a partially circumstellar origin of the $\mathrm{Rb}$ I line.

Smith \& Lambert 1989, 1990a; Smith et al. 1995). The errors in the derived $\mathrm{Li}$ abundances mainly reflect the sensitivity of our models to changes in the adopted stellar parameters. In particular, they strongly depend on the uncertainties in the determination of the effective temperature $( \pm 100-200 \mathrm{~K}$; larger for the coolest stars) and metallicity $( \pm 0.3)$, while they are less sensitive to the microturbulent velocity $\left( \pm 1 \mathrm{~km} \mathrm{~s}^{-1}\right)$, surface gravity $( \pm 0.5)$, and $F W H M( \pm 50 \mathrm{~mA})$ uncertainties. The effect of the uncertainty in the location of the pseudo-continuum around the Li I line on the measured abundances is negligible compared with any other change in the adopted stellar parameters. If we consider all these uncertainties as independent sources of error, the resulting $\mathrm{Li}$ abundances given in Table 10 are estimated to be affected by errors of the order of 0.4-0.6 dex. As an example, the changes in the derived $\mathrm{Li}$ abundance induced by slight variations of each of the atmospheric parameters used in our modelling for IRAS $15255+1944$ are shown in Table 11.

Note, however, that the estimated errors do not reflect possible non-LTE effects, dynamics of the stellar atmosphere, or errors in the model atmospheres or in the molecular/atomic linelists themselves. In particular, over-ionization and over-excitation of Li is predicted to occur under non-LTE conditions both in C-rich and O-rich AGB stars (Kiselman \& Plez 1995; Pavlenko 1996) although the effect is expected to be more pronounced in metal-deficient stars (i.e. MC AGBs). Use of LTE in Li-rich AGB stars is likely to result in underestimation of the Li abundance (Kiselman \& Plez 1995; Abia et al. 1999).

Concerning s-process elements, the non-detection of the $\mathrm{ZrO}$ molecular bands at $6474 \AA$ and $6495 \AA$ is found to be consistent with upper limits for the $\mathrm{Zr}$ abundance around $[\mathrm{Zr} / \mathrm{Fe}]=$ $0.25 \pm 0.25$ dex with respect to the solar value of $\log \varepsilon(\mathrm{Zr})=$ 2.6 dex. The synthetic spectra predict detectable $\mathrm{ZrO}$ bandheads at $6474 \AA$ and $6495 \AA$ for $\mathrm{Zr}$ abundances above $0.00-0.25 \mathrm{dex}$ for $T_{\text {eff }} \geq 3000 \mathrm{~K}$ and above $0.25-0.50$ dex for $T_{\text {eff }}<3000 \mathrm{~K}$. Actually, the fits are also reasonably good even if we do not include any $\mathrm{ZrO}$ in the synthesis. We found the same results both in the Li non-detected stars and in the Li detected ones.
This result is in strong contrast with the higher $\mathrm{Zr}$ abundances $([\mathrm{Zr} / \mathrm{Fe}]>0.5)$ found in Galactic MS-, S-stars, and in massive O-rich AGB stars in the MCs. The effect of varying the $\mathrm{Zr}$ abundance for the Li detected star IRAS 11081-4203 is displayed in Fig. 11, as an example. The best model spectrum fit for the Galactic S-star IRAS 10436-3454 is also shown in Fig. 12 for comparison. As we can see, strong $\mathrm{ZrO}$ bandheads are detected in IRAS 10436-3454, while these are completely absent from the spectrum of IRAS 11081-4203. According to our models, a modest $\mathrm{Zr}$ enhancement (with respect to the solar value) would be enough for the $\mathrm{ZrO}$ molecular bands to show up in our sample stars despite the strong veiling produced by the TiO molecule (see Fig. 11). An even lower Zr abundance is enough to produce strong $\mathrm{ZrO}$ bands in S-stars, where the $\mathrm{C} / \mathrm{O}$ ratio is $\sim 1$ because of the weaker TiO veiling, as we can see in the spectrum of IRAS 10436-3454 displayed in Fig. 12. This also favours the visibility of the $\mathrm{ZrI}$ atomic lines in the 7400-7600 and 8100-8150 ̊ spectral regions in these stars, as has already been mentioned.

\section{Discussion}

In this section, we will first try to get some information about the absolute luminosities and progenitor masses of stars in our sample. Then, we will discuss our abundance results in the framework of HBB and nucleosynthesis models and we will compare our results with the previous studies made on massive AGB stars in the MCs. Finally, we will interpret the overall results obtained in the context of stellar evolution.

\subsection{Absolute luminosities}

In constrast to the studies made in the past of AGB stars in the MCs, for which a common distance can be assumed leading to relatively well-determined absolute luminosities, the estimation of absolute luminosities for the stars in our Galactic sample is very difficult, mainly because of the large uncertainties 
Table 10. Spectroscopic $T_{\text {eff }}$ and Li abundances ${ }^{1}$ derived for the subgroup of Li detected (left) and Li non-detected stars (right).

\begin{tabular}{|c|c|c|c|c|c|}
\hline \multicolumn{3}{|c|}{ Li detected } & \multicolumn{3}{|c|}{ Li non-detected } \\
\hline IRAS name & $\begin{array}{c}T_{\text {eff }} \\
\mathrm{K}\end{array}$ & $\begin{array}{c}\log \varepsilon(\mathrm{Li}) \\
12+\log N(\mathrm{Li})\end{array}$ & IRAS name & $\begin{array}{l}T_{\text {eff }} \\
\mathrm{K}\end{array}$ & $\begin{array}{c}\log \varepsilon(\mathrm{Li}) \\
12+\log N(\mathrm{Li})\end{array}$ \\
\hline $01085+3022$ & 3300 & 2.4 & $03507+1115^{2}$ & & \\
\hline $02095-2355$ & 3300 & 1.6 & $05098-6422$ & 3000 & $<-1.0$ \\
\hline $04404-7427^{* *}$ & & $\ldots$ & $05151+6312$ & 3000 & $<+0.0$ \\
\hline $05027-2158$ & 2800 & 1.1 & $07080-5948$ & 3000 & $<+0.5$ \\
\hline $05559+3825$ & 2900 & 0.6 & $07445-2613$ & 2900 & $<-1.0$ \\
\hline $06300+6058$ & 3000 & 0.7 & $10189-3432$ & 2900 & $<-1.0$ \\
\hline $07222-2005^{* *}$ & .. & .. & $10261-5055$ & 3000 & $<-1.0$ \\
\hline 07304-2032 & 2700 & 0.9 & $13379-5426$ & 2900 & $<+0.0$ \\
\hline 09429-2148 & 3300 & 2.2 & $13442-6109^{4}$ & 3000 & $<-1.0$ \\
\hline $11081-4203$ & 3000 & 1.3 & $13475-4531$ & 2900 & $<-1.0$ \\
\hline $11525-5057$ & 3300 & 0.9 & $14086-0730^{* *}$ & $\ldots$ & $\ldots$ \\
\hline $12377-6102^{* *}$ & & & $14086-6907^{* *}$ & . & .. \\
\hline $14337-6215$ & 3300 & $2.4^{*}$ & $14247+0454$ & 2700 & $<+0.5$ \\
\hline $15211-4254$ & 3300 & 2.3 & $14266-4211$ & 2900 & $<+0.0$ \\
\hline $15255+1944$ & 2900 & 1.0 & $15193+3132$ & 2800 & $<+0.0$ \\
\hline $15576-1212$ & 3000 & 1.1 & $15586-3838$ & 3000 & $<+0.0$ \\
\hline $16030-5156$ & 3000 & 1.5 & $16037+4218$ & 2900 & $<-1.0$ \\
\hline $16260+3454$ & 3300 & 2.7 & $16503+0529$ & 2800 & $<-1.0$ \\
\hline $18025-2113^{2}$ & $\cdots$ & . & $17034-1024$ & 3300 & $<+0.0$ \\
\hline $18057-2616^{3}$ & 3000 & & $17359-2138^{* *}$ & & $\cdots$ \\
\hline $18413+1354$ & 3300 & 1.8 & $18050-2213$ & 2900 & $<-1.0$ \\
\hline $18429-1721$ & 3000 & 1.2 & $18304-0728^{2}$ & .. & .. \\
\hline $19129+2803$ & 3300 & $3.1^{*}$ & $18454-1226^{4}$ & 3300 & $<+0.0$ \\
\hline $19361-1658^{5}$ & 3000 & $1.9 / 2.0$ & $19147+5004$ & 3000 & $<+0.0$ \\
\hline $20052+0554$ & 3300 & 2.6 & 19157-1706 & 3300 & $<+0.0$ \\
\hline & & & $19412+0337$ & 3300 & $<+0.0$ \\
\hline & & & $20343-3020$ & 3000 & $<-1.0$ \\
\hline & & & $20350+3741$ & 3000 & $<+0.0$ \\
\hline & & & $22180+3225$ & 3300 & $<+0.0$ \\
\hline & & & $22466+6942$ & 3300 & $<+0.0$ \\
\hline & & & $22510+3614$ & 3000 & $<-1.0$ \\
\hline & & & $23492+0846^{4}$ & 3000 & $<-1.0$ \\
\hline
\end{tabular}

${ }^{1}$ The uncertainty in the derived Li abundances is estimated to be around $\pm 0.4-0.6$ dex.

${ }^{2}$ Possible double lined spectroscopic binary.

${ }^{3}$ The lithium line has a P cygni-type profile.

${ }^{4}$ Possible non-AGB star.

${ }^{5}$ Observed Li abundances in two different runs.

* The abundance value must be treated with some caution because the line is resolved in two components (circumstellar and stellar) and the abundance estimate corresponds to the photospheric abundance needed to fit the stellar component.

${ }^{* *}$ The $\mathrm{S} / \mathrm{N}$ ratio at $6708 \AA$ is very low to derive any reliable $T_{\text {eff }}$ and/or $\mathrm{Li}$ abundance estimate.

involved in the determination of distances within our Galaxy ${ }^{9}$. As a first approach, we can try to estimate luminosities using the period-luminosity relationship (for those sources with a well determined period). If the $\mathrm{P}-\mathrm{L}$ relationships for LMC LPVs found by Hughes \& Wood (1990) are applied to our sample of $\mathrm{OH} / \mathrm{IR}$ stars, we obtain $M_{\text {bol }}$ between $\sim-5$ and -6.2 for those stars with periods of 400-600 days while an $M_{\text {bol }}$ of $\sim-4.6$ is derived for periods around $\sim 300$ days. However, if we take those stars with the longer periods in the sample ( $\gtrsim 1000$ days), we derive $M_{\text {bol }} \lesssim-7.9$, which seems unrealistically high. This suggests that the period-luminosity relationship may not hold for the most extreme OH/IR stars (Wood et al. 1998). On the other hand, there is recent observational evidence for the existence of low metallicity (e.g. in the LMC) HBB AGB stars with luminosities brighter than the predictions of the core-mass

\footnotetext{
${ }^{9}$ Note that, in addition, AGB stars are also strongly variable. As an example, Engels et al. (1983) found an $M_{\mathrm{bol}}$ variation of 2 mag between the minimum and maximum light (from -5.0 to -7.1 mag respectively) in the Galactic $\mathrm{OH} / \mathrm{IR}$ star $\mathrm{OH} 32.8-0.3$.
}

luminosity relation that have been atributed to an excess flux from HBB (Whitelock et al. 2003). A similar effect could explain the detection of AGB stars with such high $M_{\text {bol }}$ also in our Galaxy.

\subsection{Progenitor masses}

Determining the progenitor masses of our Galactic O-rich AGB stars is not a simple task either. Several observational parameters, however, such as the $\mathrm{OH}$ maser expansion velocity or the variability period, have been proposed in the past as useful distance-independent mass indicators for this class of stars, and we will make use of them in the following.

The assumption is based on the fact that the group of OH/IR stars with longer periods and larger expansion velocities shows a Galactic distribution that corresponds to a more massive population (Baud et al. 1981; Baud \& Habing 1983; Chen et al. 2001; Jiménez-Esteban et al. 2005b) suggesting that the periods of variability and the expansion velocities of the circumstellar 


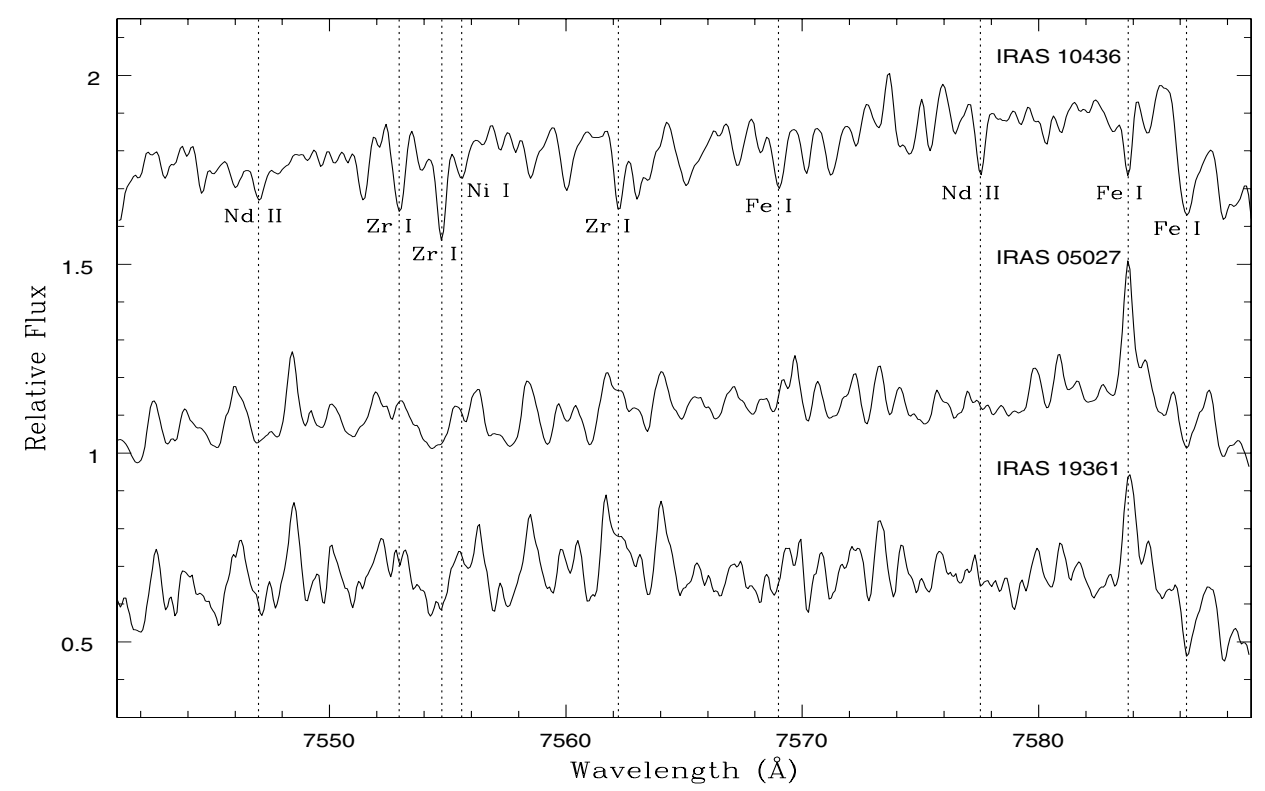

Fig. 10. High-resolution optical spectra around the $7565 \AA$ region of two stars in our sample where we show the lack of s-process element atomic lines (e.g. from $\mathrm{Zr}$ and Nd) in comparison with the Galactic S-star IRAS 10436-3459. IRAS 05027-2158 and IRAS $19361-1658$ show lithium lines with very different strength. The positions of some atomic lines of $\mathrm{Zr}$, Nd, and $\mathrm{Fe}$ are indicated. It seems that only the Fe I line at $\sim 7586 \AA$ is clearly detected in all stars.

Table 11. Sensitivity of the derived Li abundances (in dex) to slight changes in the model atmosphere parameters for IRAS $15255+1944$.

\begin{tabular}{ccc}
\hline \hline Adopted value & Change & Li abundance \\
\hline$T_{\text {eff }}=2900 \mathrm{~K}$ & $\Delta T_{\text {eff }}= \pm 100 \mathrm{~K}$ & $\Delta \log \varepsilon(\mathrm{Li})= \pm 0.3$ \\
$Z=0.0$ & $\Delta Z= \pm 0.3$ & $\Delta \log \varepsilon(\mathrm{Li})= \pm 0.2$ \\
$\xi=3 \mathrm{~km} \mathrm{~s}^{-1}$ & $\Delta \xi= \pm 1 \mathrm{~km} \mathrm{~s}^{-1}$ & $\Delta \log \varepsilon(\mathrm{Li})=\mp 0.1$ \\
$\log g=-0.5$ & $\Delta \log g=+0.5$ & $\Delta \log \varepsilon(\mathrm{Li})=+0.1$ \\
$F W H M=300 \mathrm{m \AA}$ & $\Delta F W H M= \pm 50 \mathrm{~m} \AA$ & $\Delta \log \varepsilon(\mathrm{Li})= \pm 0.1$ \\
\hline
\end{tabular}

envelopes of AGB stars must be closely correlated with the progenitor mass.

Actually, stars in our sample follow the expected trend, as is illustrated in Fig. 13. Although the limited number of stars considered prevents us from deriving any statistical conclusion based on our data alone, it seems clear that the Galactic latitude distribution of the stars in our sample becomes narrower as a function of $v_{\exp }(\mathrm{OH})$, as one would expect if the higher expansion velocities do correspond to a more massive population. A similar trend is observed when the variability period is analysed (not shown).

Figure 14 (left panel) shows the distribution of the sources in our sample on the IRAS two-colour diagram [12] - [25] vs. [25] - [60] for which $\mathrm{OH}$ expansion velocities are available. As we can see in this diagram, on average, the sources with intermediate $\mathrm{OH}$ expansion velocities, between 6 and $12 \mathrm{~km} \mathrm{~s}^{-1}$, show redder [12] $-[25]$ and [25] - [60] colours than those with low $\mathrm{OH}$ expansion velocities (i.e. below $6 \mathrm{~km} \mathrm{~s}^{-1}$ ), which implies thicker circumstellar envelopes or larger mass loss rates, but they are bluer than the subsample of stars with $\mathrm{OH}$ expansion velocities beyond $12 \mathrm{~km} \mathrm{~s}^{-1}$. Redder IRAS colours can be interpreted as the consequence of a more advanced stage on the AGB if we assume that mass loss rate increases along this evolutionary phase. Alternatively, they may also be interpreted as corresponding to a more massive population of AGB stars since in principle they should be able to develop thicker circumstellar envelopes even at a relatively early stage in their AGB evolution. A similar behaviour is observed when the variability period is analysed (Fig. 14; right panel), although the number of stars with known periods is significantly smaller. In this case we can see that the reddest sources in the diagram belong to the subgroup showing the longer periods, in excess of 700 days.

The distribution of variability periods and $\mathrm{OH}$ expansion velocities is found to be remarkably different for each of the three subgroups identified in our sample. Histograms showing the results obtained are shown in Figs. 15 and 16, respectively. The same results are presented in a different way in Table 12, where the distribution of sources among the different subtypes is presented as a function of their $\mathrm{OH}$ expansion velocity and variability properties.

In Table 12 we can see that most of the AGB stars with the lower $\mathrm{OH}$ expansion velocities $\left(v_{\exp }(\mathrm{OH})<6 \mathrm{~km} \mathrm{~s}^{-1}\right)$ are among those undetected in the Li I line $(92 \%)$ while $44 \%$ of stars with $\mathrm{OH}$ expansion velocities between 6 and $12 \mathrm{~km} \mathrm{~s}^{-1}$ (but still observable in the optical) are detected as Li-strong sources. However, there are also stars with non-detection of lithium (24\%) and stars too red or not found (32\%) within this group. Finally, a majority of the stars in the group of heavily obscured sources (too red or not found) shows $\mathrm{OH}$ expansion velocities higher than $12 \mathrm{~km} \mathrm{~s}^{-1}(66 \%)$.

Similar statistics are obtained for the variability period. Sources with relatively short periods, i.e. below 400 days, are predominantly non-Li detections (84\%), while almost half of the stars showing periods between 400 and 700 days are Li detected $(46 \%)$. Again, the same observational problems arise when we try to analyse the stars with the longer periods $(P>700$ days), since most of them are completely obscured in the optical $(88 \%)$.

\subsection{Period and $\mathrm{OH}$ expansion velocity versus $\mathrm{HBB}$}

In Fig. 17 we can see that there is actually a clear correlation between the Li abundance and the variability period in our target stars. Remarkably, for periods below 400 days $(\log P<2.6)$, only one star out of 12 is Li detected. In contrast, almost all stars 


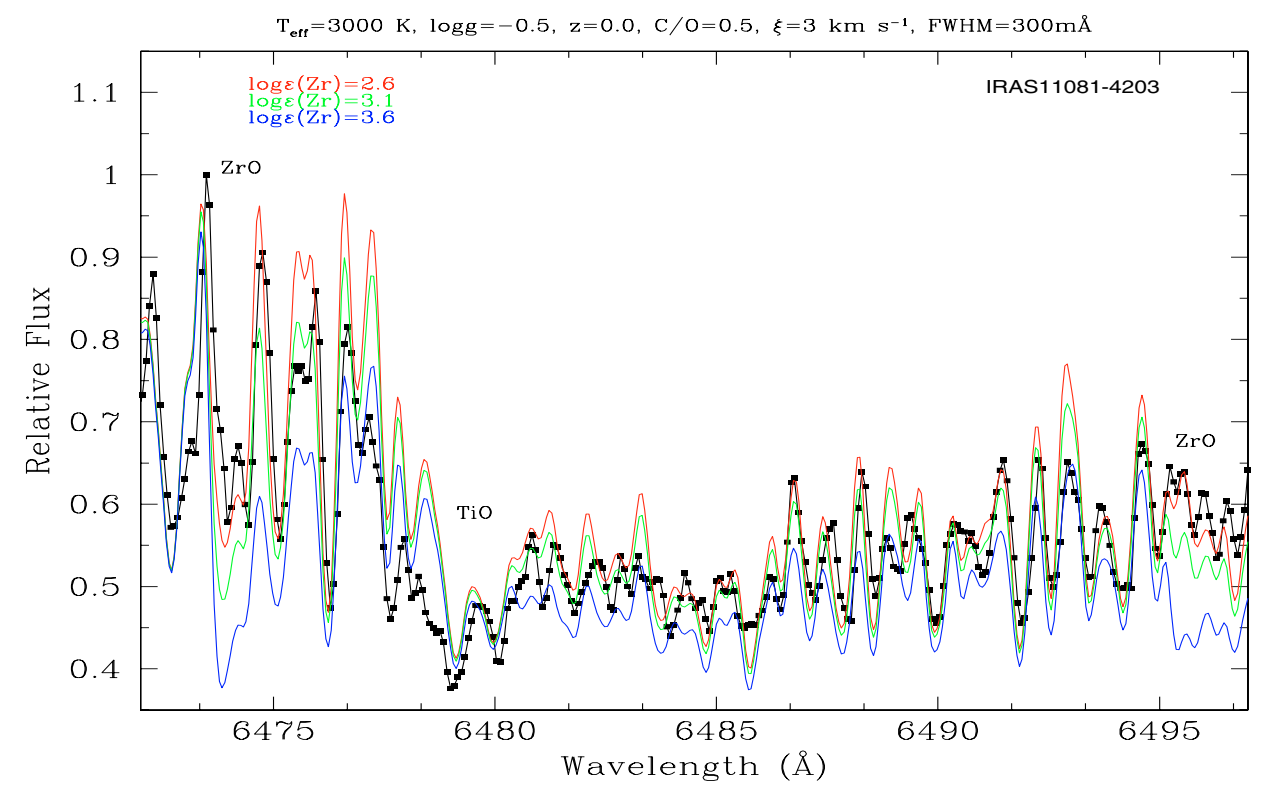

Fig. 11. Synthetic and observed spectra in the region 6460-6499 $\AA$ for the star IRAS 11081-4203, one of the Li detected stars in our sample. The synthetic spectra corresponding to $[\mathrm{Zr} / \mathrm{Fe}]=+0.0,+0.5$, and $+1.0 \mathrm{dex}($ or $\log \varepsilon(\mathrm{Zr})=2.6,3.1$, and 3.6 dex, respectively) are shown. The parameters of the best model atmosphere fit are indicated in the top label.

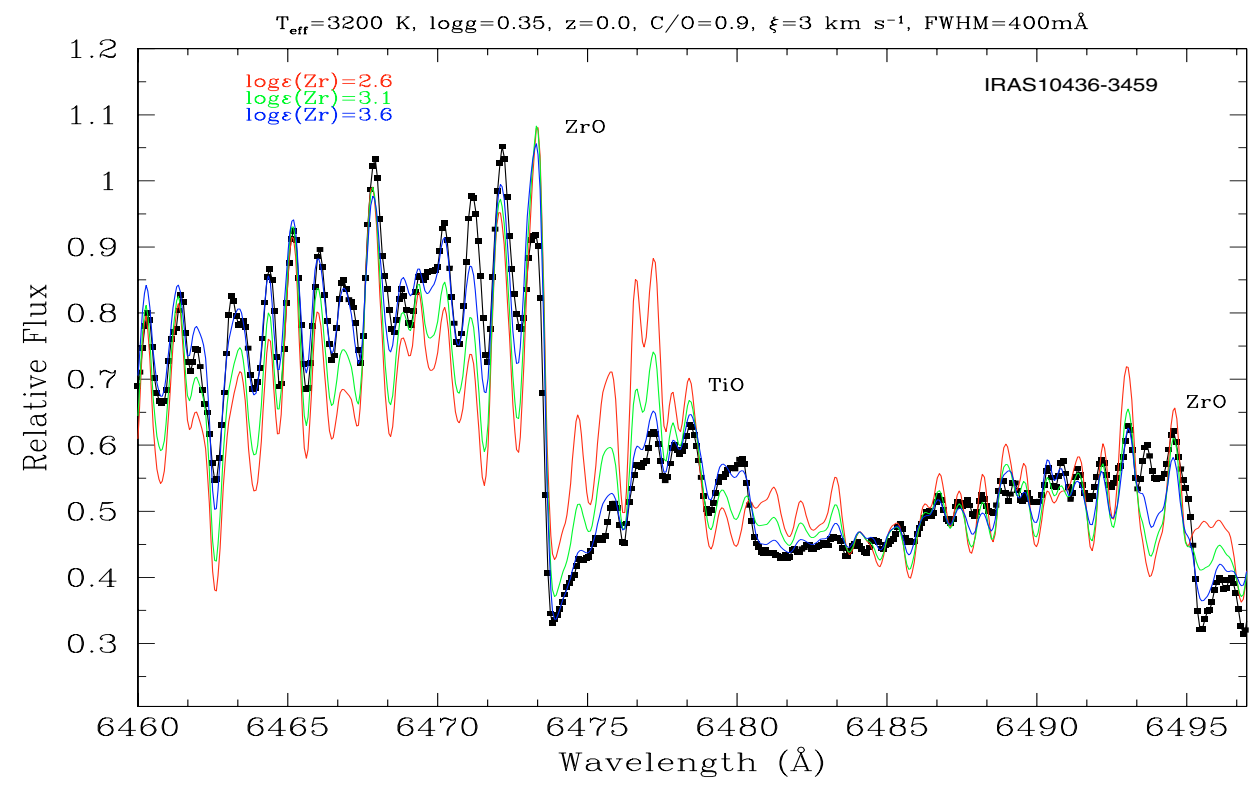

Fig. 12. Synthetic and observed spectra in the region 6460-6499 A for the Galactic S-star IRAS 10436-3454. The synthetic spectra corresponding to $[\mathrm{Zr} / \mathrm{Fe}]=+0.0,+0.5$, and $+1.0 \mathrm{dex}$ (or $\log \varepsilon(\mathrm{Zr})=2.6,3.1$, and $3.6 \mathrm{dex}$, respectively) are shown. The parameters of the best model atmosphere fit are indicated in the top label. Note the strong overabundance of $[\mathrm{Zr} / \mathrm{Fe}]=+1.0 \operatorname{dex}(\log \varepsilon(\mathrm{Zr})=3.6 \operatorname{dex})$ needed to fit the observations.

with $P>500$ days $(\log P>2.7)$ are Li-rich, the only exception being IRAS 18050-2213 (=VX Sgr), which is a peculiar, very cool semi-regular pulsating star, not showing a proper Mira variability in the last decade, which some authors identify as a massive red supergiant, and not as an AGB star (Kamohara et al. 2005).

A similar correlation, although not so clear, is found when the expansion velocities derived from the $\mathrm{OH}$ maser measurements are considered (see Fig. 18). In this case we can see that the maximum $\mathrm{Li}$ abundance increases with the $\mathrm{OH}$ expansion velocity, suggesting that the most massive AGB stars can experience a higher lithium enrichment. However, the more interesting result is the non-detection of Li in any star of the sample with $v_{\text {exp }}(\mathrm{OH})<6 \mathrm{~km} \mathrm{~s}^{-1}$. IRAS 18050-2213 is again one of the two outliers showing no Li and a very high expansion velocity, while the other one is IRAS 13442-6109, for still unknown reasons.

At present, it is generally accepted that lithium production in massive O-rich AGB stars is due to the activation of hot bottom burning at the bottom of the convective mantle, which prevents the formation of luminous C-rich AGB stars (see details in Sect. 1). If the detection of lithium is taken as a signature of $\mathrm{HBB}$ and the period and the $\mathrm{OH}$ expansion velocity are accepted as valid mass indicators, our results indicate that there are no HBB stars among the subgroup of AGB stars in our sample with 

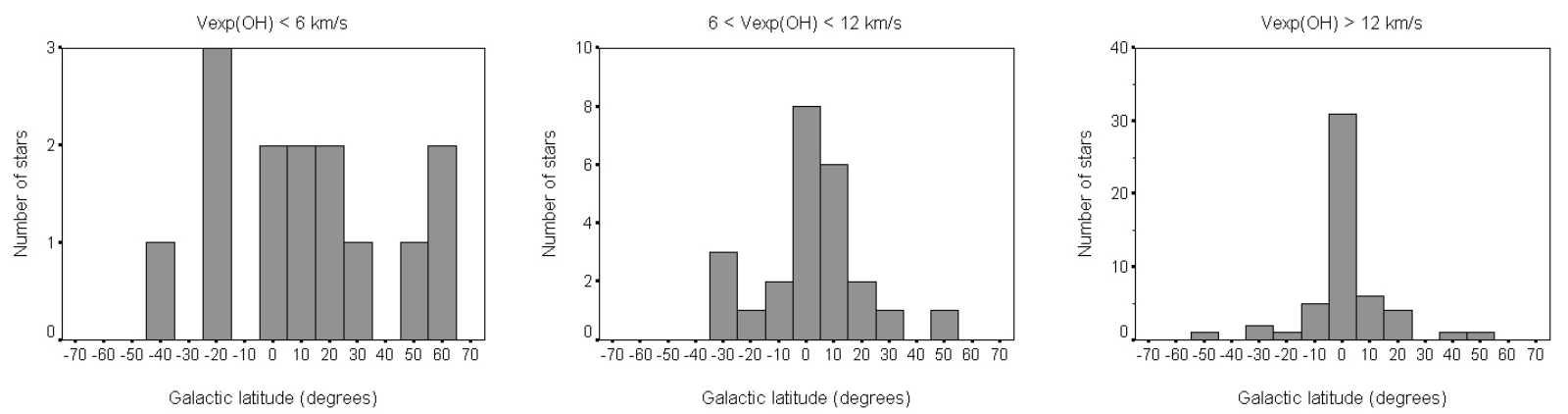

Fig. 13. Galactic latitude distribution of the sources in our sample as a function of their $\mathrm{OH}$ expansion velocities: $v_{\exp }(\mathrm{OH})<6 \mathrm{~km} \mathrm{~s}{ }^{-1}($ left panel $)$; $6<v_{\exp }(\mathrm{OH})<12 \mathrm{~km} \mathrm{~s}^{-1}$ (middle panel); and $v_{\exp }(\mathrm{OH})>12 \mathrm{~km} \mathrm{~s}^{-1}$ (right panel).
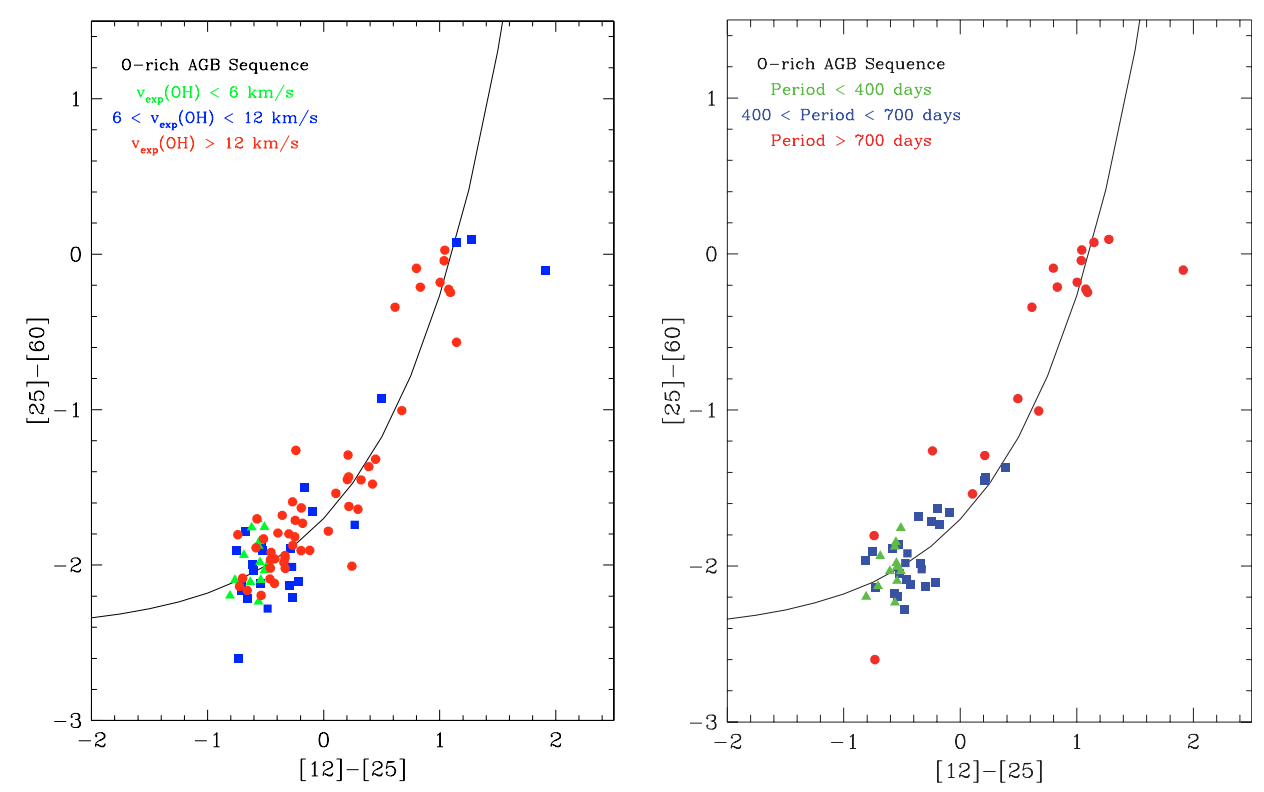

Fig. 14. Distribution of the sources included in our sample in the IRAS two-colour diagram [12]-[25] vs. [25]-[60] showing the location of the stars in our sample as a function of the $\mathrm{OH}$ expansion velocities (left panel) and variability periods (right panel). The continuum line is the "O-rich AGB sequence" (see text), which indicates the sequence of colours expected for O-rich AGB stars surrounded by envelopes with increasing thickness and/or mass loss rates (Bedijn 1987).

periods lower than 400 days and $v_{\text {exp }}(\mathrm{OH})<6 \mathrm{~km} \mathrm{~s}^{-1}$, while most of the AGB stars with periods beyond 500 days and high expansion velocities are quite probably developing HBB.

\subsection{Lithium production and HBB model predictions}

Modelling of HBB started thirty years ago with envelope models including non-instantaneous mixing coupled to the nuclear evolution (Sackmann et al. 1974). More recent models (Sackmann \& Boothroyd 1992; Mazzitelli et al. 1999, hereafter SB92 and MDV99, respectively) seem to work well in reproducing the lithium production observed in massive O-rich AGB stars in the MCs. Although the precise determination of the lithium production depends on the input physics (stellar mass, metallicity, mass loss rate, overshooting, etc.), the strongest dependence is with the convection model assumed. SB92 models predict lithium production in intermediate-mass stars assuming the so-called "mixing length theory" (MLT) framework for convection. But these models require a fine tuning of the mixing length parameter $\alpha$. MDV99 explored lithium production in intermediate-mass stars according to a more modern treatment of turbulent convection, known as the "Full Spectrum of Turbulence" (FST) model
(D’Antona \& Mazzitelli 1996, and references therein). Actually, MDV99 models are able to reproduce the strong $\mathrm{Li}$ enhancements observed in massive O-rich LMC AGB stars in a satisfactory way and provide a theoretical explanation to the existence of a few sources in the LMC with extreme luminosities of up to $M_{\mathrm{bol}}=-7.3$ and -7.6 , which are known to be long period, obscured AGB stars (Ventura et al. 2000). However, these models have not yet been tested against their Galactic analogues.

For solar metallicity, MDV99 predict lithium production as a consequence of the activation of HBB for stellar masses $\gtrsim 4.5 M_{\odot}$ without core overshooting and for $M \gtrsim 4 M_{\odot}$ including core overshooting. The amount of lithium produced during the AGB does not depend on the assumed initial lithium abundance as the star structure loses all memory of the previous history of lithium at the beginning of the AGB phase. As an example, according to this model the lithium surface abundance drops to $\log \varepsilon(\mathrm{Li}) \sim 0.3$ for a $6 M_{\odot}$ star after the second dredge-up, at the beginning of the AGB phase. If overshooting from below the convective envelope is allowed, an important decrease in the number of thermal pulses is predicted by the model and lithium production takes place before the first thermal pulse. The variation with time of the lithium abundance, total luminosity, 

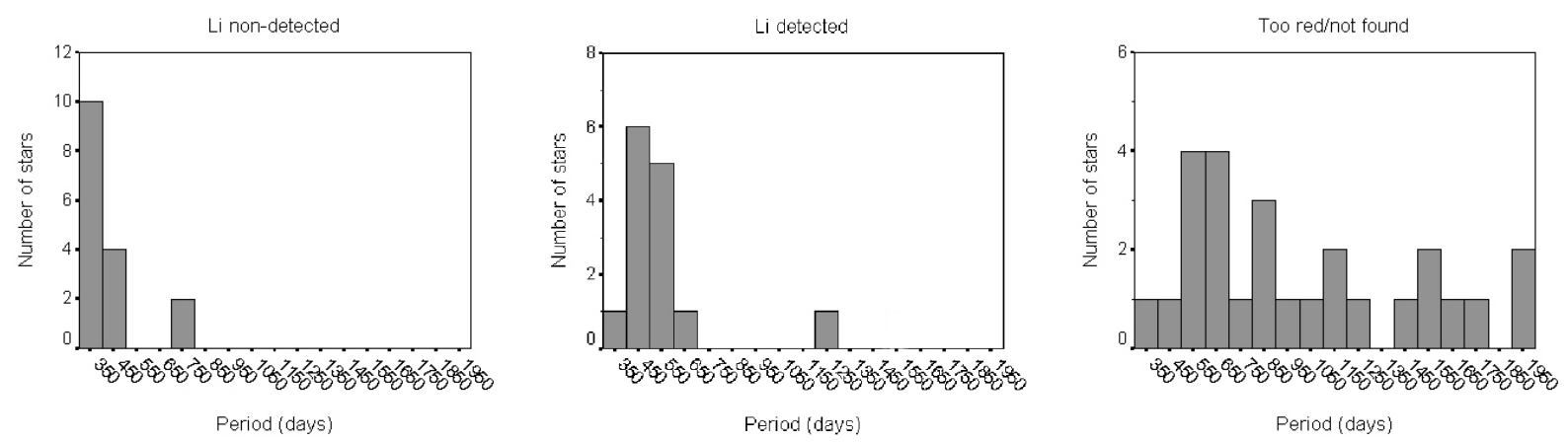

Fig. 15. Distribution of variability periods for the Li non-detected (left panel), Li detected (middle panel), and too red/not found (right panel) sources.
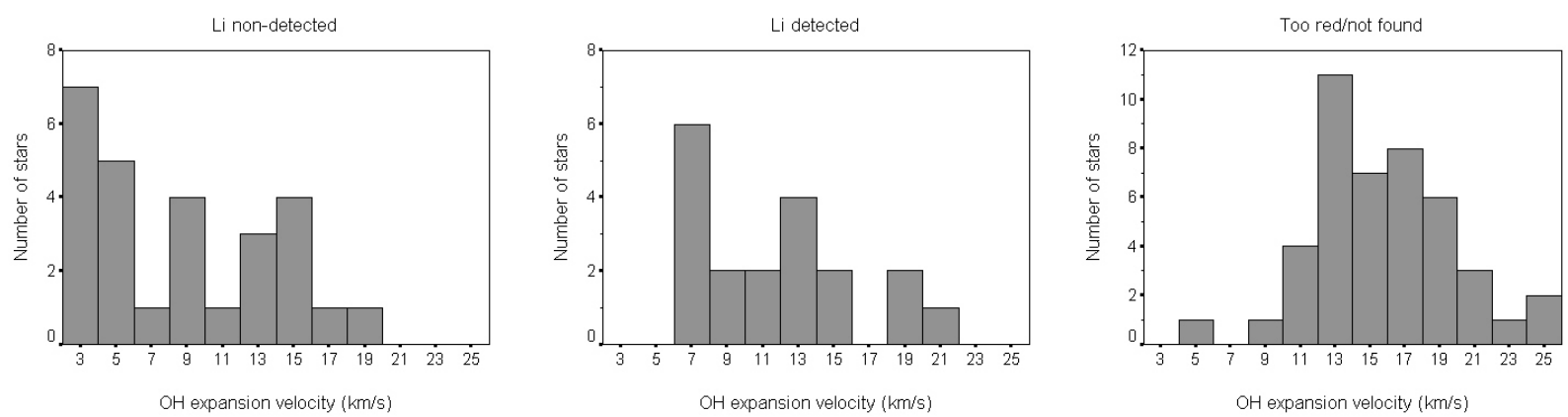

Fig. 16. Distribution of $\mathrm{OH}$ expansion velocities for the Li undetected (left panel), Li detected (middle panel), and too red/not found (right panel) sources.

Table 12. Distribution of sources among the different subgroups identified in the sample as a function of their OH expansion velocity and variability properties.

\begin{tabular}{ccccc}
\hline \hline Period (days) & Observed & Li undetected & Li detected & Too red/not found \\
\hline$<400$ & 12 & $10(84 \%)$ & $1(8 \%)$ & $1(8 \%)$ \\
$400-700$ & 26 & $4(15 \%)$ & $12(46 \%)$ & $10(39 \%)$ \\
$>700$ & 17 & $1(6 \%)$ & $1(6 \%)$ & $15(88 \%)$ \\
\hline$v_{\text {exp }}(\mathrm{OH})\left(\mathrm{km} \mathrm{s}^{-1}\right)$ & Observed & Li undetected & Li detected & Too red/not found \\
\hline$<6$ & 13 & $12(92 \%)$ & $0(0 \%)$ & $1(8 \%)$ \\
$6-12$ & 25 & $6(24 \%)$ & $11(44 \%)$ & $8(32 \%)$ \\
$>12$ & 53 & $10(19 \%)$ & $8(15 \%)$ & $35(66 \%)$ \\
\hline
\end{tabular}

and temperature at the base of the convective envelope $\left(T_{\text {bce }}\right)$ for different masses is shown in Fig. 15 of MDV99. A stronger Li-overabundance, faster increase of the luminosity at the beginning of the AGB phase, and higher $T_{\text {bce }}$ are predicted when the mass of the star is increased.

Mass loss rates and metallicities play also an important role according to MDV99. The minimum mass needed for the ignition of HBB decreases with decreasing mass loss rates. In addition, a low mass loss rate leads to a longer run of thermal pulses and to a lithium abundance which remains large for the entire thermal pulsing AGB (TP-AGB, hereafter) lifetime. In contrast, for high mass loss rates, the HBB process is stopped only after a few pulses ( $\sim 5$ for a $6 M_{\odot}$ star). Models with strong mass loss show oscillations of the $\mathrm{Li}$ abundance by orders of magnitude on short timescales of $\sim 10^{4}$ years (even within a single thermal pulse!). The temperature at the bottom of the convective envelope, $T_{\text {bce }}$, varies so much that the lithium production is temporarily stopped while the lithium already produced has already been diluted by convection. When $T_{\text {bce }}$ increases, the lithium abundance increases again. The consequence is that, at least for $20 \%$ of the time, there is negligible lithium in the envelope (at a level of approximately $\log \varepsilon(\mathrm{Li}) \lessgtr 1.0)$. This implies that the distribution of $\mathrm{Li}$ abundances derived from the observations can only be analysed in a statistical way.
On the other hand, the activation of HBB takes place at a lower mass limit of only 3.0-3.7 $M_{\odot}$ (depending on the mass loss rate considered) at the metallicity of the LMC (Ventura et al. 2000). Low metallicity models predict larger values of $T_{\text {bce }}$ and higher luminosities, and as a consequence, higher lithium production for a given stellar mass. In addition, this accelerates $\mathrm{Li}$-production and a maximum value of $\log \varepsilon(\mathrm{Li}) \sim 4.3$ is reached in a shorter timescale with respect to the solar metallicity case. The variation with time of the surface lithium abundance, stellar luminosity, and $T_{\text {bce }}$, computed for a $6 M_{\odot}$ model and different metallicities is shown in Fig. 10 of MDV99. Lower metallicity models esentially produce lithium faster due to the larger temperature at the base of the convective envelope, but show high Li abundances for a shorter time.

\subsection{Theory versus observations}

Globally considered, our results indicate that below a certain value of the period and the $\mathrm{OH}$ expansion velocity our stars do not show any Li enhancement. However, not all the stars above these values show detectable Li. The strong IR excesses associated with most of the stars in our sample suggest that they are actually at the end of their evolution as AGB stars. Surprisingly, none of the stars in our sample shows a strong s-process 


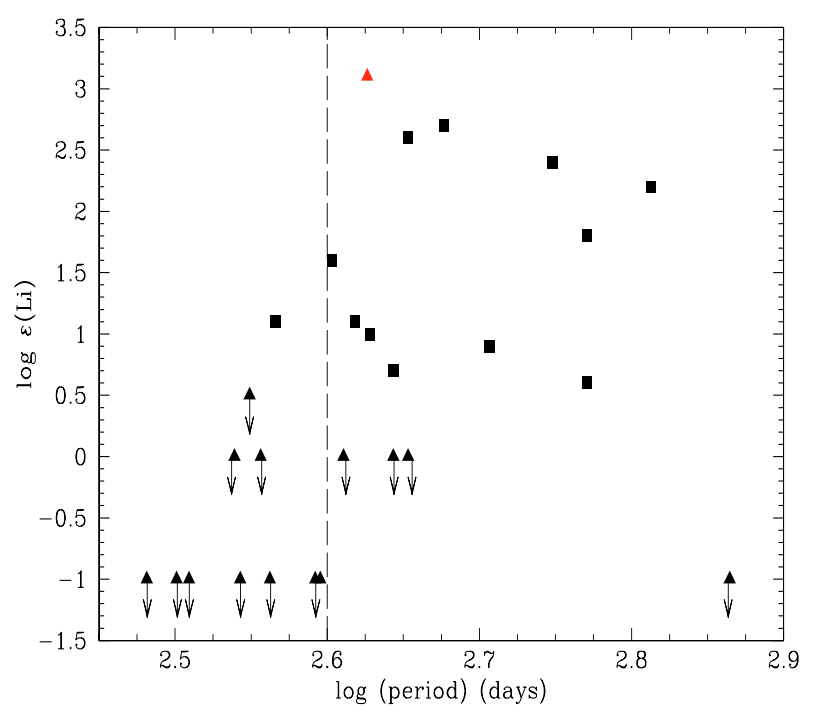

Fig. 17. Observed Li abundance vs. variability period. Upper limits to the $\mathrm{Li}$ abundance are shown with black triangles and marked with arrows. Abundance values derived from Li I lines which are resolved in two components (circumstellar and stellar) are shown with red triangles and correspond to the photospheric abundance needed to fit the stellar component. The dashed vertical line corresponds to a variability period of 400 days (see text).

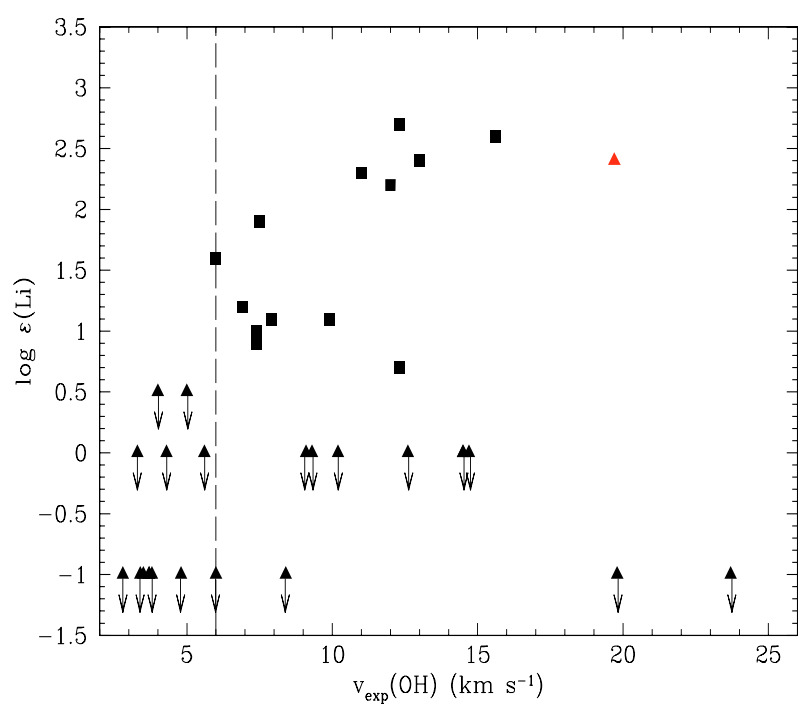

Fig. 18. Observed Li abundance vs. OH expansion velocity. Upper limits to the $\mathrm{Li}$ abundance are shown with black triangles and marked with arrows. The abundance values derived from $\mathrm{Li}$ I lines which are resolved into two components (circumstellar and stellar) are shown with red triangles and correspond to the photospheric abundance needed to fit the stellar component. The dashed vertical line corresponds to an $\mathrm{OH}$ expansion velocity of $6 \mathrm{~km} \mathrm{~s}^{-1}$ (see text).

enhancement (e.g. from Zr, Y, Sr) as would have been expected if they were stars that have experienced a significant third dredgeup during their AGB evolution. In the case of the more massive stars in our sample one could argue that the mass loss is so strong at the high metallicity of our Galaxy (compared to the MC metallicity) that their AGB lifetime may become shortened significantly, so that they will end this phase experiencing too few thermal pulses before they completely lose their envelope. However, the lack of s-process enhancement is also observed in the group of stars in which no lithium has been detected.
In order to interpret these results, several scenarios can be proposed:

i) all the stars in the sample are HBB stars, and thus, considerably massive $\left(M \gtrsim 4 M_{\odot}\right)$. The difference between the Li detected and the Li undetected ones would be just that the undetected ones are in a very early stage as AGB stars. This hypothesis would explain the absence of s-process enhancement (e.g. Zr, Y, Sr) in all the stars in the sample, but it would not be consistent with the non-detection of lithium in the group of stars with the shorter periods and lower $\mathrm{OH}$ expansion velocities. HBB models predict a considerable Li enhancement just after the arrival at the AGB. Statistically, there should be no difference in the behaviour of the Li abundances for any star in the sample, and this is not observed.

ii) the sample is composed by a combination of low-mass $(M \lesssim$ $\left.1.5 M_{\odot}\right)$ and high-mass $\left(M \gtrsim 4 M_{\odot}\right)$ stars. In this scenario, the optically bright O-rich AGB stars in our sample with the shorter periods and lower $\mathrm{OH}$ expansion velocities, undetected in $\mathrm{Li}$, would belong to the population of lowmass stars in the Galaxy that do not experience HBB and evolve all the way along the AGB as O-rich stars. However, these stars are expected to evolve very slowly and develop a large number of thermal pulses during the AGB. At the end of their AGB evolution they are expected to become strongly enhanced in s-process elements as a consequence of the dredge-up of processed material induced by the many thermal pulses experienced. But the Li undetected stars in our sample do not show this enhancement in s-process elements. The only way to reconcile this result with their status as low-mass stars would imply assuming that they are at a very early stage as AGB stars, which again seems to be incompatible with the strong IR excess detected by IRAS. Such a strong excess is only expected in low-mass stars at the very end of the AGB. Alternatively, they could experience less thermal pulses than predicted by the models or the dredge-up process could be very inefficient. But this is not what we observe in other low- and intermediate-mass stars in the Galaxy, such as the S-stars and many C-rich stars, which show the strong s-process enhancement that is not observed in our stars. Therefore, we must conclude that the hypothesis of these Li undetected stars being identified as low-mass AGB stars seems to be implausible.

iii) all the stars in the sample are intermediate-mass stars $(M \gtrsim$ $3 M_{\odot}$ ) but only a fraction of them, the more massive ones $\left(M \gtrsim 4 M_{\odot}\right)$, experience HBB. This scenario would explain why the sources with lower periods and $\mathrm{OH}$ expansion velocities in our sample are undetected in the Li I line, while at the same time, they do not show any s-process enhancement. These stars, although slightly below the minimum mass needed to develop HBB, would also experience a very strong mass loss rate and only a few thermal pulses $(\sim 5-10)$ before the early termination of the TP-AGB as a consequence of the strong mass loss. The initial envelope of these stars would also be quite massive and thus a slow change of the $\mathrm{C} / \mathrm{O}$ ratio is expected because of the huge dilution of the dredged up material. But how can we explain the large spread of Li abundances observed in the group of sources in our sample with longer periods and larger $\mathrm{OH}$ expansion velocities? These are all supposed to be HBB stars. And HBB models predict a large fluctuation in the Li abundance as the star evolves along the TP-AGB (see Fig. 15 of MDV99 again) between two consecutive pulses. Actually, the timescale of the Li production phase in the TP-AGB is 
predicted to be of the order or slightly smaller than the timescale between pulses $\left(\sim 10^{4}\right.$ years for a massive star with $\left.M \gtrsim 4 M_{\odot}\right)$. In addition, as the AGB evolution proceeds, the surface Li abundances decrease again very rapidly as a consequence of the ${ }^{3} \mathrm{He}$ becoming almost completely burned in the envelope, and thus, the Li production ceases (e.g. MDV99; Forestini \& Charbonnel 1997). This would be consistent with the detection of strong $\mathrm{Li}$ abundances in $\sim 50 \%$ of the stars in our sample for which we suspect that HBB is active. In this scenario, the strongly obscured sources for which we were not able to obtain an optical spectrum could also be interpreted as a population of high mass stars developing HBB with even more extreme observational properties. They are sources with longer periods ( $>700$ days) and larger $\mathrm{OH}$ expansion velocities $\left(>12 \mathrm{~km} \mathrm{~s}^{-1}\right)$ which may have developed optically thick circumstellar envelopes as a consequence of the huge mass loss experienced in the AGB. These stars must also be detectable as Li-rich at least for some time during their AGB evolution but, unfortunately, they are so strongly reddened in the optical range that their study is only possible in the infrared, where no strong Li lines are present. We propose that the $\mathrm{HBB}$ nature of these obscured stars can be elucidated by determining their ${ }^{12} \mathrm{C} /{ }^{13} \mathrm{C}$ ratios, which can also be used as an HBB indicator, through near infrared spectroscopy.

\subsection{Comparison with the O-rich AGB stars in the MCs}

The situation is quite different for the AGB stars in the MCs. In contrast with their Galactic analogues, the more luminous AGB stars in the MCs (with $-7 \leq M_{\text {bol }} \leq-6$ ) are O-rich stars showing s-process element enhancements. In addition, a higher proportion of them ( $\sim 80 \%$ compared to $\sim 50 \%$ in our Galaxy) also shows lithium overabundances. The enrichment in lithium indicates that they are also HBB stars, but why are these stars also enriched in s-process elements?

The answer to this question must be related to the different metallicity of the stars in the MCs with respect to our Galaxy. Actually, theoretical models predict a higher efficiency of the dredge-up in low metallicity atmospheres (e.g. Herwig 2004) with respect to those with solar metallicity (e.g. Lugaro et al. 2003).

Moreover, there is observational evidence that lower metallicity environments are also less favourable to dust production, as is suggested by the smaller number of heavily obscured AGB stars in the MCs (Trams et al. 1999; Groenewegen et al. 2000), compared to our Galaxy. This is supported by the lower dust-to-gas ratios derived by van Loon (2000) in the few obscured AGB stars in the MCs for which this analysis has been made.

If mass loss is driven by radiation pressure on the dust grains, it might be less efficient with decreasing metallicity (Willson 2000). In that case, longer AGB lifetimes would be expected, which could increase the chance of nuclear-processed material reaching the stellar surface. This would also explain the larger proportion of luminous C-rich stars (up to $M_{\text {bol }} \sim-6$ ) observed in the MCs (Plez et al. 1993; Smith \& Lambert 1989, 1990a; Smith et al. 1995) with respect to the Galaxy. The slow evolution predicted for AGB stars in the MCs as a consequence of the less efficient mass loss leaves time for more thermal pulses to occur during the AGB lifetime and, therefore, a more effective dredgeup of s-process elements to the surface can be expected before the envelope is completely gone at the end of the AGB. This would explain why even the more massive stars in the MCs show a strong s-process enrichment in contrast to their Galactic counterparts. In our Galaxy the only AGB stars showing a similar overabundance in s-process elements seem to be the result of the evolution of low- to intermediate-mass stars $\left(M \lesssim 1.5-2.0 M_{\odot}\right)$, while no, or very little, s-process enhancement is observed in Galactic AGB stars with higher main sequence masses.

In addition, the lower critical mass (which also decreases with decreasing mass loss rate, see Sect. 5.4) needed to develop HBB $\left(M \gtrsim 3 M_{\odot}\right.$ at the metallicity of the LMC, compared to the $\sim 4 M_{\odot}$ limit in our Galaxy) favours the simultaneous detection of s-process elements and $\mathrm{Li}$ enrichment in a larger number of AGB stars in the MCs. In our Galaxy, the Lirich sample of AGB stars is restricted to the fraction of stars with main sequence masses $M \gtrsim 4 M_{\odot}$. In contrast to their MC counterparts, these stars evolve so rapidly (because of the strong mass loss) that there is no time for a significant enhancement in s-process elements, as the results here presented seems to demonstrate.

Obviously, more refined s-process calculations taking into account the effects of HBB and mass loss, using a large grid of stellar evolutionary models with different masses and metallicities are strongly encouraged.

\subsection{Evolutionary connections}

The well known existence of at least two different chemical branches of AGB stars (C-rich and O-rich) and the dependence of this chemical segregation on the main sequence mass and on the environmental conditions in which these stars evolve (such as the metallicity) is a long debated issue in stellar evolution. Moreover, the connection with the chemical composition observed in Galactic post-AGB stars and/or well evolved PNe (which are expected to be the result of the evolution of AGB stars with a wide variety of masses) has never been addressed in detail from an overall perspective.

Stars classified as post-AGB in the literature are mainly lowmass stars, according to their wide Galactic distribution. They are thought to represent the fraction of stars which evolve so slowly that they are still visible for some time as stars with intermediate spectral types before they become PNe. Many of these stars are C-rich and show strong s-process element enhancements [s-process/Fe] +1.5 (van Winckel \& Reyniers 2000; Reddy et al. 1999; Klochkova et al. 1999), in agreement with what we would expect if they were the final product of the evolution of low-mass AGB stars. However, other post-AGB stars do not show the characteristic signatures of the third dredge-up. In the non-enriched sources the s-process element abundances can be as low as $[\mathrm{Zr} / \mathrm{Fe}] \lesssim 0.2$ (Luck et al. 1990; van Winckel 1997). These objects might be stars with even lower masses ( $M \sim 0.8-1.0 M_{\odot}$ ) which have evolved off the AGB before the third dredge-up could occur (Marigo et al. 1999). Some of these sources are high Galactic latitude, hot B-type post-AGB stars (e.g. Conlon et al. 1993; Mooney et al. 2001) that show strong carbon deficiencies. In the more extreme cases they will probably never become PNe.

On the other hand, and as we have shown in this article, the more massive AGB stars may also show a similar lack of sprocess elements, but in this case as a consequence of the rapid evolution induced by the strong mass loss. These stars are more difficult to observe in the optical as they are expected to evolve as heavily obscured sources from the TP-AGB to the PN stage but may be the progenitors of most Galactic PNe.

Actually, a similar chemical segregation is also observed in Galactic PNe. Classically, they are classified as type I, II or III 
as a function of their chemical abundance properties (Peimbert 1978). They are expected to cover a wide range of progenitor masses and thus show different chemical signatures as a consequence of their previous passage along the AGB.

In particular, the higher mass fraction of stars (with $M>$ $4 M_{\odot}$ ) identified in this paper as stars developing HBB and showing a strong lithium enhancement are expected to arrive at the PN stage still as heavily obscured stars. These stars would evolve into N-rich type I PNe, which are characterized by their large $\mathrm{He}$ abundances $(\mathrm{He} / \mathrm{H}>0.10)$ and $\mathrm{N} / \mathrm{O}$ ratios (Manchado 2003, 2004; Mampaso 2004, and references therein). They are thought to represent the fraction of the more massive PNe in the Galaxy, as is confirmed by their strong concentration at low Galactic latitudes. Their strong N overabundances would be consistent with their identification as PNe resulting from the evolution of HBB AGB stars.

\section{Conclusions}

As a result of a high-resolution spectroscopic survey carried out in the optical for a large sample (102) of massive Galactic O-rich AGB stars, we have detected the Li I resonance line at $6708 \AA$ in 25 stars, while 32 stars did not display this Li I line. The rest of the stars observed ( 45 stars) were heavily obscured by their thick circumstellar envelopes and, as such, they were too red or were not found at optical wavelengths.

We have classified the sample into three subgroups on the basis of their $\mathrm{OH}$ expansion velocity and variability period. From their relative distribution in the IRAS two-colour diagram [12] [25] vs. [25] - [60] and Galactic distribution, we conclude that they must represent populations of Galactic O-rich AGB stars with different progenitor masses.

By combining MARCS model atmospheres and synthetic spectroscopy with extensive line lists we were able to derive the fundamental stellar parameters ( $T_{\text {eff }}, \log g, \mathrm{C} / \mathrm{O}, \xi$, etc.) and obtain the $\mathrm{Li}$ and $\mathrm{Zr}$ abundances in those stars for which an optical spectrum had been obtained. Our chemical abundance analysis shows that half of stars show $\mathrm{Li}$ overabundances in the range between $\log \varepsilon(\mathrm{Li}) \sim+0.5$ and +3.0 dex. This is interpreted as a signature of the activation of $\mathrm{HBB}$, confirming that they are actually massive AGB stars $\left(M>4 M_{\odot}\right)$. However, these stars do not show any zirconium enhancement (taken as representative of the s-process element enrichment), indicating that they behave differently from the more massive (and luminous) AGB stars in the MCs.

Assuming that the variability period and the $\mathrm{OH}$ expansion velocity can be taken as distance-independent mass indicators and by comparing our results with the theoretical predictions of $\mathrm{HBB}$ and nucleosynthesis models, we conclude that the O-rich AGB stars in our sample are all very massive $\left(M>3 M_{\odot}\right)$, even those non-detected in lithium; but only the more massive ones $\left(M>4 M_{\odot}\right.$; with periods longer than 400 days and $v_{\text {exp }}(\mathrm{OH})>6 \mathrm{~km} \mathrm{~s}^{-1}$ ) experience HBB. The lack of lithium enrichment found in some of the sources thought to belong to the group of more massive AGB stars in the sample is explained as (i) the consequence of the timescale of the $\mathrm{Li}$ production phase being of the order of or slightly smaller than the interpulse time ( $\sim 10^{4}$ years) or, alternatively, (ii) by the ${ }^{3} \mathrm{He}$ exhaustion in the envelope interrupting the $\mathrm{Li}$ production, in agreement with the predictions made by the models.

The group of stars displaying the most extreme observational properties (with periods sometimes longer than 700 days and $\left.v_{\exp }(\mathrm{OH})>12 \mathrm{~km} \mathrm{~s}^{-1}\right)$ are believed to represent the more massive AGB stars in our Galaxy. Unfortunately, these stars are strongly obscured by their thick circumstellar envelopes so we could not carry out any type of analysis in the optical range. This very high-mass population should also experience HBB and show strong Li enhancement but no s-process element overabundances. We propose that the HBB status of these obscured stars can be determined by measuring their ${ }^{12} \mathrm{C} /{ }^{13} \mathrm{C}$ ratios in the nearinfrared, which can also be used as an HBB indicator.

Our results suggest that the dramatically different abundance pattern found in AGB stars belonging to the MCs and to our Galaxy can be explained in terms of the different metallicity conditions under which these stars evolved. This constitutes strong observational evidence that the chemical evolution of massive AGB stars is strongly modulated by metallicity.

Globally considered, our results can also be used to establish evolutionary connections between TP-AGB stars, postAGB stars, and PNe. In particular, we suggest that the HBB AGB stars identified in our sample must be the precursors of N-rich type I PNe.

Acknowledgements. DAGH is grateful to J.I. González-Hernández from the Instituto de Astrofísica de Canarias for the adaptation of his $\chi^{2}$ code used in this work. AM and PGL acknowledge support from grant AYA 2004-3136 and AYA 2003-9499 from the Spanish Ministerio de Educación y Ciencia.

\section{References}

Abia, C., \& Isern, J. 1996, ApJ, 460, 443

Abia, C., \& Isern, J. 1997, MNRAS, 289, L11

Abia, C., \& Isern, J. 2000, ApJ, 536, 438

Abia, C., \& Wallerstein, G. 1998, MNRAS, 293, 89

Abia, C., Boffin, H. M. J., Isern, J., \& Rebolo, R. 1991, A\&A, 245, L1

Abia, C., Boffin, H. M. J., Isern, J., \& Rebolo, R. 1993, A\&A, 272, 455

Abia, C., Pavlenko, Y., \& de Laverny, P. 1999, A\&A, 351, 273

Abia, C., Busso, M., Gallino, R., et al. 2001, ApJ, 559, 1117

Allard, F., Hauschildt, P. H., \& Schwenke, D. 2000, ApJ, 540, 1005

Alvarez, R., \& Plez, B. 1998, A\&A, 330, 1109

Alvarez, R., Lancon, A., Plez, B., \& Wood, P. R. 2000, A\&A, 353, 322

Aringer, B., Kerschbaum, F., \& Jrgensen, U. G. 2002, A\&A, 395, 915

Baud, B., \& Habing, H. J. 1983, A\&A, 127, 73

Baud, B., Habing, H. J., Matthews, H. E., \& Winnberg, A. 1981, A\&A, 95, 156

Bedijn, P. J. 1987, A\&A, 186, 136

Beichman, G., Neugebauer, H. J., Habing, P. E., Clegg, T. J., \& Chester, eds. 1988, Infrared Astronomical Satellite (IRAS) Catalogs and Atlases Explanatory Supplement, NASA RP-1190

Boffin, H. M. J., Abia, C., Isern, J., \& Rebolo, R. 1993, A\&AS, 102, 361

Busso, M., Gallino, R., \& Wasserburg, G. J. 1999, ARA\&A, 37, 239

Busso, M., Gallino, R., Lambert, D. L., Travaglio, C., \& Smith, V. V. 2001, ApJ, 557,802

Cameron, A. G. W., \& Fowler, W. A. 1971, ApJ, 164, 111

Cenarro, A. J., Gorgas, J., Cardiel, N., Vazdekis, A., \& Peletier, R. F. 2002, MNRAS, 329, 863

Charbonnel, C. 2005, in Cosmic Abundances as Records of Stellar Evolution and Nucleosynthesis, ed. T. G. Barnes III, \& F. N. Bash, ASPC, 336, 119

Chen, P. S., Szczerba, R., Kwok, S., \& Volk, K. 2001, A\&A, 368, 1006

Chengalur, J. N., Lewis, B. M., Eder, J., \& Terzian, Y. 1993, ApJS, 89, 189

Conlon, E. S., Dufton, P. L., McCausland, R. J. H., \& Keenan, F. P. 1993, ApJ, 408,593

D'Antona, F., \& Mazzitelli, I. 1996, ApJ, 470, 1093

Decin, L., Vandenbussche, B., Waelkens, C., et al. 2003a, A\&A, 400, 679

Decin, L., Vandenbussche, B., Waelkens, C., et al. 2003b, A\&A, 400, 709

Engels, D., Kreysa, E., Schultz, G. V., \& Sherwood, W. A. 1983, A\&A, 124, 123 Forestini, M., \& Charbonnel, C. 1997, A\&AS, 123, 241

Gallino, R., Busso, M., Lugaro, M., Travaglio, C., \& Straniero, O. 2000, in Proc. of the 35th Liege International Astrophysics Colloquium, ed. A. Noels et al. (Liège: Institut d'Astrophysique), 81

García-Hernández, D. A., Abia, C., García-Lario, P., \& Manchado, A. 2006a, A\&A, 452, 1049

García-Hernández, D. A., García-Lario, P., Plez, B., et al. 2006b, Science, 314, 1751

García-Lario, P. 1992, Ph.D. Thesis, La Laguna University

Grevesse, N., \& Sauval, A. J. 1998, SSRv, 85, 161

Groenewegen, M. A. T., \& de Jong, T. 1998, A\&A, 337, 797 
Groenewegen, M. A. T., Blommaert, J. A. D. L., Cioni, M.-R., et al. 2000, Mem. Soc. Astron. Ital., 71, 639

Groenewegen, M. A. T., Sevenster, M., Spoon, H. W. W., \& Pérez, I. 2002, A\&A, 390,511

Gustafsson, B., Edvardsson, B., Eriksson, K., et al. 2003, Modelling of Stellar Atmospheres, ed. N. Piskunov, W. W. Weiss, \& D. F. Gray, ASP, IAU Symp., 210, A4

Herman, J., \& Habing, H. J. 1985, A\&AS, 59, 523

Herwig, F. 2004, ApJ, 605, 425

Hinkle, K., Wallace, L., Valenti, J., \& Harmer, D. 2000, Visible and Near Infrared Atlas of the Arcturus Spectrum 3727-9300 Å, ed. K. Hinkle, L. Wallace, J. Valenti, \& D. Harmer (San Francisco: ASP)

Höfner, S., Gautschy-Loidl, R., Aringer, B., \& Jrgensen, U. G. 2003, A\&A, 399, 589

Holweger, H., \& Müller, E. A. 1974, Sol. Phys., 39, 19

Hughes, S. M. G., \& Wood, P. R. 1990, AJ, 99, 784

Jiménez-Esteban, F. M., Agudo-Mérida, L., Engels, D., \& García-Lario, P. 2005a, A\&A, 431, 779

Jiménez-Esteban, F. M., García-Lario, P., \& Engels, D. 2005b, in Planetary Nebulae as Astronomical Tools, ed. R. Szczerba, G. Stasińska, \& S. K. Górny, AIP Conf. Proc., 804, 141

Jiménez-Esteban, F. M., García-Lario, P., Engels, D., \& Perea Calderón, J. V. 2006a, A\&A, 446, 773

Jiménez-Esteban, F. M., García-Lario, P., Engels, D., \& Manchado, A. 2006b, A\&A, 458, 533

Jones, T. J., Bryja, C. O., Gehrz, R. D., et al. 1990, ApJS, 74, 785

Kamohara, R., Deguchi, S., Miyoshi, M., \& Shen, Z. Q. 2005, PASJ, 57, 341

Kastner, J. H., Forveille, T., Zuckerman, B., \& Omont, A. 1993, A\&A, 275, 163

Kholopov, P. N., Samus, N. N., Frolov, M. S., et al. 1998, GCVS, Combined General Catalogue of Variable Stars

Kiselman, D., \& Plez, B. 1995, MmSAI, 66, 429

Klochkova, V. G., Szczerba, R., Panchuk, V. E., \& Volk, K. 1999, A\&A, 345, 905

Kupka, F., Piskunov, N. E., Ryabchikova, T. A., Stempels, H. C., \& Weiss, W. W. 1999, A\&AS, 138, 119

Kurucz, R. L. 1993, SAO, Cambridge (CDROM 18)

Kurucz, R. L. 1994, SAO, Cambridge (CDROM 20-22)

Kwok, S., Volk, K., \& Bidelman, W. P. 1997, ApJS, 112, 557

Lambert, D. L., Smith, V. V., Busso, M., Gallino, R., \& Straniero, O. 1995, ApJ, 450,302

Lattanzio, J. C. 2003, Planetary Nebulae: Their Evolution and Role in the Universe, ed. S. Kwok, M. Dopita, \& R. Sutherland, ASP, IAU Symp., 209, 73

Lattanzio, J. C., \& Lugaro, M. 2005, in Nuclei in the Cosmos VIII, Nucl. Phys. A, 758, 477

Le Bertre, T. 1993, A\&AS, 97, 729

Levesque, E. M., Massey, P., Olsen, K. A. G., et al. 2005, ApJ, 628, 973

Lewis, B. M., Eder, J., \& Terzian, Y. 1990, ApJ, 362, 634

Lewis, B. M. 1994, ApJS, 93, 549

Lewis, B. M., David, P., \& Le Squeren, A. M. 1995, A\&AS, 111, 237

Lockwood, G. W. 1985, ApJS, 58, 167

Loidl, R., Lancon, A., \& Jrgensen, U. G. 2001, A\&A, 37, 1065

Luck, R. E., \& Lambert, D. L. 1982, ApJ, 256, 189

Luck, R. E., Bond, H. E., \& Lambert, D. L. 1990, ApJ, 357, 188

Lugaro, M., Herwig, F., Lattanzio, J. C., Gallino, R., \& Straniero, O. 2003, ApJ, 586,1305

Mampaso, A. 2004, in Asymmetrical Planetary Nebulae III: Winds, Structure and the Thunderbird, ed. M. Meixner, J. H. Kastner, B. Balick, \& N. Soker, ASP Conf. Proc., 313, 265

Manchado, S. 2003, Planetary Nebulae: Their Evolution and Role in the Universe, ed. S. Kwok, M. Dopita, R. Sutherland, ASP, IAU Symp., 209, 431

Manchado, A. 2004, in Asymmetrical Planetary Nebulae III: Winds, Structure and the Thunderbird, ed. M. Meixner, J. H. Kastner, B. Balick, \& N. Soker, ASP Conf. Proc., 313, 3

Marigo, P., Girardi, L., \& Bressan, A. 1999, A\&A, 344, 123
Mazzitelli, I., D’Antona, F., \& Ventura, P. 1999, A\&A, 348, 846

Mooney, C. J., Rolleston, W. R. J., Keenan, F. P., et al. 2001, MNRAS, 326, 1101

Moore, C. E., Minnaert, M. G. J., \& Hootgast, J. 1966, The solar spectrum, $2935 \AA ̊$ to $8770 \AA ̊$, NBS Monog. 61 (Washington, DC: NBS)

Mowlavi, N. 1999, A\&A, 344, 617

Mowlavi, N. 2002, Cosmic Chemical Evolution, ed. K. Nomoto, \& J. W. Truran (Kluwer Academic Publishers), IAU Symp., 187, 57

Nakashima, J., Jiang, B. W., Deguchi, S., Sadakane, K., \& Nakada, Y. 2000, PASJ, 52, 275

Neckel, H. 1999, Sol. Phys., 184, 421.

Nyman, L.-A., Booth, R. S., Carlstrom, U., et al. 1992, A\&AS, 93, 121

Pavlenko, Y. 1996, in Cool stars, stellar systems, and the sun, ed. R. Pallavicini, \& A. K. Dupree, ASP Conf. Ser., 109, 569

Peimbert, M. 1978, in Planetary nebulae (Dordrecht: D. Reidel. Publishing Co), 215

Plez, B. 1990, MmSAI, 61, 765

Plez, B. 1998, A\&A, 337, 495

Plez, B., Brett, J. M., \& Nordlund, Å. 1992, A\&A, 256, 551

Plez, B., Smith, V. V., \& Lambert, D. L. 1993, ApJ, 418, 812

Plez, B., van Eck, S., Jorissen, A., Edvardsson, B., Eriksson, K., \& Gustafsson, B. 2003, Modelling of Stellar Atmospheres, ed. N. Piskunov, W. W. Weiss, \& D. F. Gray, ASP, IAU Symp., 210, A2

Reddy, B. E., Bakker, E. J., \& Hrivnak, B. J. 1999, ApJ, 524, 831

Sackmann, I.-J., \& Boothroyd, A. I. 1992, ApJ, 392, L71

Sackmann, I. J., Smith, R. L., \& Despain, K. H. 1974, ApJ, 187, 555

Sevenster, M. N., Chapman, J. M., Habing, H. J., Killeen, N. E. B., \& Lindqvist, M. 1997, A\&AS, 122, 79

Sivagnanam, P., Le Squeren, A. M., Foy, F., \& Tran Minh, F. 1989, A\&A, 211, 341

Slootmaker, A., Habing, H. J., \& Herman, J. 1985, A\&AS, 59, 465

Smith, V. V., \& Lambert, D. L. 1985, ApJ, 294, 326

Smith, V. V., \& Lambert, D. L. 1989, ApJ, 345, L75

Smith, V. V., \& Lambert, D. L. 1990a, ApJ, 361, L69

Smith, V. V., \& Lambert, D. L. 1990b, ApJS, 72, 387

Smith, V. V., Plez, B., Lambert, D. L., \& Lubowich, D. A. 1995, ApJ, 441, 735

Straniero, O., Gallino, R., Busso, M., et al. 1995, ApJ, 440, L85

Straniero, O., Chieffi, A., Limongi, M., et al. 1997, ApJ, 478, 332

Straniero, O., Limongi, M., Chieffi, A., et al. 2000, MmSAI, 71, 719

te Lintel Hekkert, P., Versteege-Hensel, H. A., Habing, H. J., \& Wiertz, M. 1989, A\&AS, 78, 399

te Lintel Hekkert, P., Caswell, J. L., Habing, H. J., Haynes, R. F., \& Norris, R. P. 1991, A\&AS, 90, 327

Trams, N. R., van Loon, J. Th., Waters, L. B. F. M., et al. 1999, A\&A, 346, 843 Vaglio, P., Gallino, R., Busso, M., et al. 1999, in Nuclei in the Cosmos V, ed. N. Prantzos, \& S. Harissopulos (Paris: Éditions Frontières), 223

van Loon, J. Th. 2000, A\&A, 354, 125

van Loon, J. Th., Cioni, M.-R. L., Zijlstra, A. A., \& Loup, C. 2005, A\&A, 438, 273

van Winckel, H. 1997, A\&A, 319, 561

van Winckel, H., \& Reyniers, M. 2000, A\&A, 354, 135

Vanture, A. D., \& Wallerstein, G. 2002, ApJ, 564, 395

Ventura, P., D'Antona, F., \& Mazzitelli, I. 2000, A\&A, 363, 605

Wallace, L., Hinkle, K., \& Livingston, W. 1993, An atlas of the photospheric spectrum from 8900 to $13600 \mathrm{~cm}^{-1}$ (7350 to $11230 \AA$ A), NSO Technical Report 93-001, Tucson: National Solar Observatory

Wallace, L., Hinkle, K., \& Livingston, W. 1998, An atlas of the spectrum of the solar photosphere from 13500 to $28000 \mathrm{~cm}^{-1}$ (3570 to $7405 \AA$ A)", Tucson, AZ: National Optical Astronomy Observatories

Whitelock, P. A., Menzies, J., Feast, M., et al. 1994, MNRAS, 267, 711

Whitelock, P. A., Feast, M. W., van Loon, J. Th., \& Zijlstra, A. A. 2003, MNRAS, 342, 86

Willson, L. A. 2000, ARA\&A, 38, 573

Winters, J. M., Le Bertre, T., Jeong, K. S., Helling, Ch., \& Sedlmayr, E. 2000, A\&A, 361, 641

Wood, P. R., Bessell, M. S., \& Fox, M. W. 1983, ApJ, 272, 99

Wood, P. R., Habing, H. J., \& McGregor, P. J. 1998, A\&A, 336, 925 
D. A. García-Hernández et al.: Li and Zr in massive Galactic O-rich AGBs, Online Material $p 1$

\section{Online Material}


D. A. García-Hernández et al.: Li and Zr in massive Galactic O-rich AGBs, Online Material p 2

Table 1. The sample of Galactic O-rich AGB stars.

\begin{tabular}{|c|c|c|c|c|c|c|c|}
\hline IRAS name & Other names & \multicolumn{2}{|c|}{ Galactic Coordinates } & Variability & Spectral Type & {$[12]-[25]$} & {$[25]-[60]$} \\
\hline $01037+1219$ & WX Psc & 128.64 & -50.11 & Mira & M10 & -0.192 & -1.632 \\
\hline $01085+3022$ & AW Psc & 127.96 & -32.04 & Mira & M9 & -0.331 & -2.021 \\
\hline $01304+6211$ & V669 Cas & 127.81 & -0.02 & Mira & & +0.494 & -0.927 \\
\hline $02095-2355$ & IRC-20029 & 206.68 & -71.55 & & M6 & -0.748 & -1.992 \\
\hline $02316+6455$ & V656 Cas & 133.61 & +4.46 & Mira & M8 & -0.463 & -2.089 \\
\hline $03507+1115$ & IK Tau & 177.95 & -31.41 & Mira & M9e & -0.725 & -2.137 \\
\hline $04404-7427$ & SY Men & 286.97 & -34.74 & Mira & & -0.297 & -2.132 \\
\hline $05027-2158$ & T Lep & 222.67 & -32.71 & Mira & M7e & -0.706 & -2.129 \\
\hline $05073+5248$ & NV Aur & 156.44 & +7.83 & Mira & M10 & +0.204 & -1.450 \\
\hline $05098-6422$ & U Dor & 274.30 & -35.16 & Mira & M7e & -0.510 & -1.756 \\
\hline $05151+6312$ & BW Cam & 148.28 & +14.57 & Mira & M9 & -0.698 & -2.084 \\
\hline $05559+3825$ & V373 Aur & 173.24 & +7.27 & Mira & M9-10 & -0.563 & -2.174 \\
\hline $06297+4045$ & IRC+40156 & 174.11 & +14.12 & & M8 & -0.094 & -1.658 \\
\hline $06300+6058$ & AP Lyn & 154.31 & +21.52 & Mira & M7+ & -0.357 & -1.680 \\
\hline $07080-5948$ & NSV 3436 & 270.50 & -21.14 & $\ldots$ & & -0.631 & -2.109 \\
\hline $07222-2005$ & & 234.58 & -2.16 & . & M9 & -0.733 & -2.600 \\
\hline 07304-2032 & Z Pup & 235.90 & -0.69 & Mira & M5e & -0.752 & -1.908 \\
\hline $07445-2613$ & SS Pup & 242.43 & -0.69 & Mira & M6e & -0.807 & -2.197 \\
\hline 09194-4518 & MQ Vel & 269.09 & +3.10 & Mira & $\ldots$ & -0.273 & -2.012 \\
\hline 09429-2148 & IW Hya & 255.80 & +23.35 & Mira & M9 & -0.217 & -2.106 \\
\hline 10189-3432 & V Ant & 271.03 & +18.61 & Mira & M7e & -0.561 & -2.235 \\
\hline $10261-5055$ & VZ Vel & 281.31 & +5.57 & SRa & M6e & -0.542 & -2.096 \\
\hline $11081-4203$ & & 283.71 & +16.75 & $\ldots$ & $\mathrm{Me}$ & -0.699 & -1.895 \\
\hline $11525-5057$ & & 294.00 & +10.65 & & $\cdots$ & -0.299 & -1.964 \\
\hline $12377-6102$ & & 301.63 & +1.52 & Mira & .. & -0.195 & -1.907 \\
\hline $12384-4536$ & & 301.08 & +16.94 & $\ldots$ & $\ldots$ & -0.120 & -1.905 \\
\hline $13203-5536$ & & 307.45 & +6.71 & $\ldots$ & $\ldots$ & -0.249 & -1.818 \\
\hline $13328-6244$ & & 308.00 & -0.56 & $\cdots$ & $\ldots$ & +0.295 & -1.641 \\
\hline $13341-6246$ & & 308.14 & -0.63 & $\mathrm{~L}$ & $\ldots$ & -0.300 & -1.799 \\
\hline $13379-5426$ & & 310.14 & +7.48 & $\ldots$ & $\ldots$ & -0.290 & -1.893 \\
\hline 13442-6109 & OH $309.6+0.7$ & 309.62 & +0.73 & & M10 & -0.337 & -1.774 \\
\hline $13475-4531$ & V618 Cen & 313.60 & +15.88 & Mira & $\mathrm{Me}$ & -0.764 & -2.096 \\
\hline $13517-6515$ & & 309.51 & -3.46 & & & +0.040 & -1.782 \\
\hline $14086-0730$ & IO Vir & 334.78 & +50.12 & Mira & M8 & -0.333 & -1.938 \\
\hline $14086-6907$ & & 310.04 & -7.61 & & & -0.333 & -1.955 \\
\hline $14247+0454$ & RS Vir & 352.67 & +57.97 & Mira & M5e & -0.553 & -1.847 \\
\hline $14266-4211$ & & 321.67 & +16.84 & $\cdots$ & $\ldots$ & -0.547 & -2.121 \\
\hline $14337-6215$ & & 314.87 & -2.09 & $\cdots$ & $\ldots$ & -0.518 & -1.831 \\
\hline 15099-5509 & RAFGL 4212 & 322.26 & +2.10 & & & -0.267 & -1.873 \\
\hline $15193+3132$ & V S CrB & 49.47 & +57.17 & Mira & M6e & -0.510 & -2.034 \\
\hline $15211-4254$ & & 330.43 & +11.47 & & & -0.609 & -1.997 \\
\hline $15255+1944$ & WX Ser & 29.51 & +53.48 & Mira & M8e & -0.483 & -2.280 \\
\hline $15303-5456$ & & 324.83 & +0.69 & . & $\ldots$ & +0.421 & -1.479 \\
\hline $15576-1212$ & FS Lib & 358.44 & +29.52 & Mira & M8-9 & -0.535 & -1.860 \\
\hline $15586-3838$ & NSV 7388 & 338.79 & +10.48 & $\cdots$ & $\ldots$ & -0.672 & $-1,785$ \\
\hline $16030-5156$ & V352 Nor & 330.50 & +0.01 & Mira & M4e & -0.438 & -1.914 \\
\hline $16037+4218$ & V1012 Her & 66.88 & +48.00 & $\cdots$ & M8 & -0.549 & -1.981 \\
\hline $16260+3454$ & V 697 Her & 56.37 & +43.53 & Mira & M9 & -0.245 & -1.712 \\
\hline $16503+0529$ & RX Oph & 23.69 & +28.78 & Mira & M5 & -0.687 & -1.936 \\
\hline $17034-1024$ & V850 Oph & 10.68 & +17.76 & Mira & M8 & -0.543 & -2.007 \\
\hline $17103-0559$ & & 15.57 & +18.70 & $\cdots$ & $\ldots$ & -0.539 & -2.195 \\
\hline $17239-2812$ & & 352.61 & -0.18 & $\ldots$ & $\ldots$ & -0.268 & -1.593 \\
\hline
\end{tabular}


Table 1. continued.

\begin{tabular}{|c|c|c|c|c|c|c|c|}
\hline IRAS name & Other names & \multicolumn{2}{|c|}{ Galactic Coordinates } & Variability & Spectral Type & $\overline{[112]-[25]}$ & {$[25]-[60]$} \\
\hline $17359-2138$ & & 5.40 & +5.09 & $\cdots$ & M7 & -0.705 & -2.163 \\
\hline $17433-1750$ & GLMP 637 & 9.56 & +5.61 & $\ldots$ & $\ldots$ & +1.144 & -0.567 \\
\hline $17433-2523$ & & 3.10 & +1.68 & $\ldots$ & $\ldots$ & -0.421 & -1.960 \\
\hline $17443-2519$ & RAFGL 5386 & 3.29 & +1.52 & $\ldots$ & $\ldots$ & +0.322 & -1.452 \\
\hline $17501-2656$ & V4201 Sgr & 2.58 & -0.43 & SR & M8-9 & -0.239 & -1.262 \\
\hline $18025-2113$ & IRC-20427 & 8.93 & -0.01 & & $\mathrm{cM} 4$ & -0.167 & -1.503 \\
\hline $18050-2213$ & VX Sgr & 8.34 & -1.00 & $\mathrm{SRc}$ & M4eIa & -0.740 & -1.805 \\
\hline $18057-2616$ & SAO 186357 & 4.89 & -3.18 & $\ldots$ & M & -0.575 & -1.703 \\
\hline $18071-1727$ & OH $12.8+0.9$ & 12.75 & +0.89 & $\ldots$ & & +1.275 & +0.094 \\
\hline $18083-2630$ & NSV 24316 & 4.97 & -3.73 & $\ldots$ & M6 & +0.243 & -2.007 \\
\hline $18172-2305$ & & 8.95 & -3.89 & $\ldots$ & $\ldots$ & -0.459 & -2.020 \\
\hline $18198-1249$ & OH $18.3+0.4$ & 18.30 & +0.43 & $\ldots$ & $\ldots$ & +1.092 & -0.246 \\
\hline $18257-1000$ & V441 Sct & 21.46 & +0.49 & Mira & $\ldots$ & +1.038 & -0.042 \\
\hline $18273-0738$ & V436 Sct & 23.73 & +1.23 & Mira & $\ldots$ & +0.105 & -1.538 \\
\hline $18276-1431$ & V445 Sct & 17.68 & -2.03 & Mira & 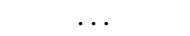 & +1.914 & -0.103 \\
\hline $18304-0728$ & IRC-10434 & 24.21 & +0.63 & .. & M5r & -0.394 & -1.794 \\
\hline $18312-1209$ & & 20.18 & -1.70 & $\ldots$ & & +0.447 & -1.319 \\
\hline $18314-1131$ & NSV 24500 & 20.76 & -1.46 & $\ldots$ & M6 & -0.533 & -1.750 \\
\hline $18348-0526$ & V437 Sct & 26.54 & +0.62 & Mira & & +0.615 & -0.341 \\
\hline $18413+1354$ & V837 Her & 44.56 & +8.02 & Mira & M8III & -0.424 & -2.118 \\
\hline $18429-1721$ & V3952 Sgr & 16.84 & -6.60 & Mira & M9 & -0.527 & -1.907 \\
\hline $18432-0149$ & V1360Aql & 30.71 & +0.42 & Mira & $\ldots$ & +0.799 & -0.091 \\
\hline $18437-0643$ & V440 Sct & 26.42 & -1.93 & Mira & & +0.389 & -1.367 \\
\hline $18454-1226$ & IRC-10463 & 21.53 & -4.92 & $\ldots$ & M6 & -0.685 & -2.306 \\
\hline $18460-0254$ & V1362 Aql & 30.09 & -0.68 & Mira & $\ldots$ & +1.003 & -0.181 \\
\hline $18488-0107$ & V1363 Aql & 31.99 & -0.49 & Mira & $\ldots$ & +1.043 & +0.026 \\
\hline $18549+0208$ & OH $35.6-0.3$ & 35.57 & -0.34 & & $\cdots$ & +0.833 & -0.212 \\
\hline $18560+0638$ & V1366 Aql & 39.71 & +1.50 & Mira & $\ldots$ & +0.210 & -1.292 \\
\hline 19059-2219 & V3880 Sgr & 14.66 & -13.61 & Mira & M8 & -0.343 & -1.984 \\
\hline $19129+2803$ & & 60.67 & +7.74 & & & -0.521 & -2.045 \\
\hline $19147+5004$ & TZ Cyg & 81.22 & +16.80 & $\mathrm{Lb}$ & M6 & -0.659 & -2.163 \\
\hline 19157-1706 & RAFGL 2361 & 20.53 & -13.55 & $\ldots$ & $\ldots$ & -0.458 & -1.965 \\
\hline $19161+2343$ & RAFGL 2362 & 57.12 & +5.12 & $\ldots$ & $\ldots$ & +0.217 & -1.623 \\
\hline $19192+0922$ & NSV 24761 & 44.79 & -2.31 & $\ldots$ & $\ldots$ & +0.215 & -1.433 \\
\hline $19254+1631$ & GLMP 915 & 51.80 & -0.22 & $\ldots$ & $\ldots$ & +1.077 & -0.226 \\
\hline $19361-1658$ & NSV 24833 & 22.74 & -17.93 & $\ldots$ & M8 & -0.268 & -2.207 \\
\hline $19412+0337$ & V1415 Aql & 42.34 & -9.86 & Mira & M9 & -0.583 & -1.889 \\
\hline $19426+4342$ & & 77.48 & +9.73 & $\ldots$ & $\ldots$ & -0.659 & -2.216 \\
\hline $20052+0554$ & V1416 Aql & 47.37 & -13.95 & Mira & M9 & -0.453 & -1.919 \\
\hline $20077-0625$ & V1300 Aql & 36.36 & -20.42 & Mira & $\mathrm{M}$ & -0.182 & -1.731 \\
\hline $20109+3205$ & V557 Cyg & 70.47 & -1.08 & Mira & M7 & -0.562 & -1.876 \\
\hline $20181+2234$ & V371 Vul & 63.44 & -7.72 & Mira & $\ldots$ & +0.268 & -1.741 \\
\hline $20272+3535$ & GLMP 999 & 75.27 & -1.84 & & & +1.147 & +0.074 \\
\hline $20343-3020$ & RT Mic & 13.59 & -34.91 & Mira & & -0.605 & -2.033 \\
\hline $20350+3741$ & V1828 Cyg & 77.90 & -1.84 & Mira & M7 & -0.814 & -1.961 \\
\hline $22177+5936$ & NSV 25875 & 104.91 & +2.41 & $\ldots$ & $\ldots$ & +0.673 & -1.006 \\
\hline $22180+3225$ & YZ Peg & 89.60 & -20.24 & $\ldots$ & $\ldots$ & -0.620 & -1.758 \\
\hline $22466+6942$ & & 112.69 & +9.59 & $\ldots$ & $\ldots$ & -0.472 & -1.981 \\
\hline $22510+3614$ & NSV 14347 & 97.90 & -20.56 & $\ldots$ & M7 & -0.567 & -1.705 \\
\hline $23492+0846$ & DO 8089 & 99.10 & -51.03 & $\ldots$ & M5 & -0.716 & -1.900 \\
\hline
\end{tabular}


Table 2. The sample of comparison stars.

\begin{tabular}{llrrcccc}
\hline \hline IRAS name & Other names & \multicolumn{2}{l}{ Galactic Coordinates } & Variability & Spectral Type & {$[12]-[25]$} & {$[25]-[60]$} \\
\hline $00213+3817$ & R And & 117.07 & -23.98 & Mira & S5/4.5e & -0.723 & -2.104 \\
$02192+5821$ & S Per & 134.62 & -2.19 & SRc & M4eIa & -0.407 & -1.897 \\
$10436-3459$ & Z Ant & 275.86 & +20.94 & SR & S5.4 & -0.830 & -2.172 \\
$18397+1738$ & V821 Her & 47.78 & +10.01 & Mira & Ce & -0.873 & -1.499 \\
$19133-1703$ & T Sgr & 20.32 & -13.01 & Mira & S5/6e & -1.125 & -1.372 \\
$20166+3717$ & WX Cyg & 75.43 & +0.87 & Mira & C9,2eJ & -1.191 & +1.445 \\
$23416+6130$ & PZ Cas & 115.06 & -0.05 & SRc & M3vIa & +0.070 & -1.538 \\
$23554+5612$ & WY Cas & 115.57 & -5.62 & Mira & S6/6e & -0.633 & -1.286 \\
$23587+6004$ & WZ Cas & 116.76 & -1.91 & SRc & SC7/10e & -1.280 & -1.348 \\
\hline
\end{tabular}

Table 3. The sample of peculiar carbon AGB stars.

\begin{tabular}{llrrcccc}
\hline \hline IRAS name & Other names & \multicolumn{2}{c}{ Galactic Coordinates } & Variability & Spectral Type & {$[12]-[25]$} & {$[25]-[60]$} \\
\hline $00247+6922$ & V668 Cas & 120.86 & +6.87 & Mira & $\mathrm{C}$ & -0.774 & -1.762 \\
$04130+3918$ & RAFGL 6312 & 160.41 & -8.09 & $\ldots$ & $\mathrm{C}$ & -1.273 & -1.633 \\
$09425-6040$ & GLMP 260 & 282.04 & -5.88 & $\ldots$ & $\mathrm{C}$ & +0.790 & -1.045 \\
$17297+1747$ & V833 Her & 40.80 & +25.30 & Mira & $\mathrm{C}$ & -0.340 & -1.859 \\
$19321+2757$ & V1965 Cyg & 62.57 & +3.96 & Mira & $\mathrm{C}$ & -0.703 & -1.598 \\
$19594+4047$ & V1968 Cyg & 76.52 & +5.59 & Mira & $\mathrm{C}$ & -0.285 & -1.594 \\
$20072+3116$ & V1969 Cyg & 69.35 & -0.87 & Mira & $\mathrm{C}$ & -0.644 & -1.483 \\
$23166+1655$ & LL Peg & 93.53 & -40.35 & Mira & $\mathrm{C}$ & +0.101 & -1.238 \\
$23320+4316$ & LP And & 108.46 & -17.15 & Mira & $\mathrm{C}$ & -0.777 & -1.555 \\
\hline
\end{tabular}

Table 4. Log of the spectroscopic observations.

\begin{tabular}{cccccc}
\hline \hline Run & Telescope & Instrumentation & Dates & $\begin{array}{c}\text { Dispersion } \\
(\AA / \text { pix })\end{array}$ & $\begin{array}{c}\text { Spectral Range } \\
(\AA)\end{array}$ \\
\hline$\# 1$ & $4.2 \mathrm{~m}$ WHT & UES & 16-17 August 1996 & 0.065 & $5300-9400$ \\
$\# 2$ & 4.2 m WHT & UES & 10-12 June 1997 & 0.065 & $4700-10300$ \\
$\# 3$ & $4.2 \mathrm{~m}$ WHT & UES & 18-20 August 1997 & 0.065 & $4700-10300$ \\
$\# 4$ & 3.6 m ESO & CASPEC & 21-24 February 1997 & 0.085 & $6000-8200$ \\
\hline
\end{tabular}


D. A. García-Hernández et al.: Li and Zr in massive Galactic O-rich AGBs, Online Material p 5

Table 5. Galactic O-rich AGB stars where the lithium line was detected.

\begin{tabular}{lccccc}
\hline \hline IRAS name & Run & $\begin{array}{c}v_{\text {exp }}(\mathrm{OH}) \\
\left(\mathrm{km} \mathrm{s}^{-1}\right)\end{array}$ & Ref. & $\begin{array}{c}\text { Period } \\
\text { (days) }\end{array}$ & Ref. \\
\hline $01085+3022$ & 3 & 13.0 & 3 & 560 & 1 \\
$02095-2355$ & 1 & $\ldots$ & $\ldots$ & $\ldots$ & $\ldots$ \\
$04404-7427^{*}$ & 4 & 7.7 & 1 & 534 & 2 \\
$05027-2158$ & 4 & 7.9 & 1 & 368 & 1 \\
$05559+3825$ & 3 & single peak & 4 & 590 & 3 \\
$06300+6058$ & 3 & 12.3 & 2 & 440 & 4 \\
$07222-2005^{*}$ & 4 & 8.2 & 1 & 1200 & 10 \\
$07304-2032$ & 4 & 7.4 & 5 & 509 & 1 \\
$09429-2148$ & 4 & 12.0 & 2 & 650 & 1 \\
$11081-4203$ & 4 & single peak & 1 & $\ldots$ & $\ldots$ \\
$11525-5057$ & 4 & single peak & 1 & $\ldots$ & $\ldots$ \\
$12377-6102^{*}$ & 4 & 20.4 & 1 & $\ldots$ & $\ldots$ \\
$14337-6215$ & 4 & 19.7 & 9 & $\ldots$ & $\ldots$ \\
$15211-4254$ & 4 & 11.0 & 1 & $\ldots$ & $\ldots$ \\
$15255+1944$ & 1 & 7.4 & 2 & 425 & 1 \\
$15576-1212$ & 4 & 9.9 & 2 & 415 & 1 \\
$16030-5156$ & 4 & single peak & 1 & $\ldots$ & $\ldots$ \\
$16260+3454$ & 1 & 12.3 & 2 & 475 & 1 \\
$18025-2113$ & 1 & 10.6 & 1 & $\ldots$ & $\ldots$ \\
$18057-2616$ & 1 & 18.7 & 1 & $\ldots$ & $\ldots$ \\
$18413+1354$ & 2 & 14.7 & 4 & 590 & 3 \\
$18429-1721$ & 1 & 6.9 & 1 & $\ldots$ & $\ldots$ \\
$19129+2803$ & 2 & single peak & 1 & 420 & 10 \\
$19361-1658$ & 2,3 & 7.5 & 1 & $\ldots$ & $\ldots$ \\
$20052+0554$ & 2 & 15.6 & 12 & 450 & 3 \\
\hline
\end{tabular}

* Tentative Li detection.

References for the $\mathrm{OH}$ expansion velocities:

1) te Lintel Hekkert et al. (1991); 2) te Lintel Hekkert et al. (1989); 3) Chengalur et al. (1993); 4) Slootmaker et al. (1985); 5) Sivagnanam et al. (1989); 6) Lewis et al. (1990); 7) Lewis et al. (1995); 8) Groenewegen et al. (2002) (from CO data); 9) Sevenster et al. (1997); 10) Groenewegen \& de Jong (1998); 11) Nyman et al. (1992); 12) Lewis (1994) .

References for the periods:

1) Combined General Catalogue of Variable Stars (GCVS), Kholopov (1998); 2) Whitelock et al. (1994); 3) Jones et al. (1990); 4) Lockwood (1985); 5) Engels et al. (1983); 6) Nakashima et al. (2000); 7) Slootmaker et al. (1985); 8) Le Bertre (1993); 9) Herman \& Habing (1985); 10) Jiménez-Esteban et al. (2006b).
Table 6. Galactic O-rich AGB stars where the lithium line was not detected.

\begin{tabular}{lccccc}
\hline \hline IRAS name & Run & $\begin{array}{c}v_{\text {exp }}(\mathrm{OH}) \\
\left(\mathrm{km} \mathrm{s}^{-1}\right)\end{array}$ & Ref. $^{1}$ & $\begin{array}{c}\text { Period } \\
\text { (days) }\end{array}$ & Ref. $^{{ }^{1}}$ \\
\hline $03507+1115$ & 3 & 16.5 & 2 & 470 & 1 \\
$05098-6422$ & 4 & 6.0 & 2 & 394 & 2 \\
$05151+6312$ & 1 & 14.5 & 2 & $\ldots$ & 3 \\
$07080-5948$ & 4 & 5.0 & 1 & $\ldots$ & $\ldots$ \\
$07445-2613$ & 4 & 4.8 & 2 & 391 & 1 \\
$10189-3432$ & 4 & 3.4 & 2 & 303 & 1 \\
$10261-5055$ & 4 & 3.7 & 1 & 317 & 1 \\
$13379-5426$ & 4 & 10.2 & 1 & $\ldots$ & $\ldots$ \\
$13442-6109^{* *}$ & 4 & 23.7 & 1 & $\ldots$ & $\ldots$ \\
$13475-4531$ & 4 & 3.7 & 1 & $\ldots$ & $\ldots$ \\
$14086-0730^{*}$ & 4 & 13.7 & 2 & $\ldots$ & $\ldots$ \\
$14086-6907^{*}$ & 4 & 13.4 & 1 & $\ldots$ & $\ldots$ \\
$14247+0454$ & 4 & 4.0 & 2 & 354 & 1 \\
$14266-4211$ & 4 & 9.1 & 1 & $\ldots$ & $\ldots$ \\
$15193+3132$ & 1 & 3.3 & 2 & 360 & 1 \\
$15586-3838$ & 4 & 9.3 & 1 & $\ldots$ & $\ldots$ \\
$16037+4218$ & 1 & 3.5 & 1 & 360 & 10 \\
$16503+0529$ & 2 & 3.8 & 3 & 323 & 1 \\
$17034-1024$ & 2 & single peak & 1 & 346 & 1 \\
$17359-2138^{*}$ & 2 & 8.5 & 1 & $\ldots$ & $\ldots$ \\
$18050-2213$ & 3 & 19.8 & 2 & 732 & 1 \\
$18304-0728$ & 3 & 15.3 & 2 & $\ldots$ & $\ldots$ \\
$18454-1226 * *$ & 3 & single peak & 1 & $\ldots$ & $\ldots$ \\
$19147+5004$ & 1 & 12.6 & 1 & $\ldots$ & $\ldots$ \\
$19157-1706$ & 2 & 14.7 & 1 & $\ldots$ & $\ldots$ \\
$19412+0337$ & 2 & 14.5 & 6 & 440 & 3 \\
$20343-3020$ & 3 & 8.4 & 1 & 349 & 2 \\
$20350+3741$ & 3 & $\ldots$ & $\ldots$ & 450 & 3 \\
$22180+3225$ & 1 & 4.3 & 3 & $\ldots$ & $\ldots$ \\
$22466+6942$ & 1 & 5.6 & 1 & 408 & 6 \\
$22510+3614$ & 1 & 2.8 & 3 & $\ldots$ & $\ldots$ \\
$23492+0846^{* *}$ & 1 & $\ldots$ & $\ldots$ & $\ldots$ & $\ldots$ \\
\hline & & & & & \\
\hline
\end{tabular}

${ }^{*}$ Poor $\mathrm{S} / \mathrm{N}$ ratio.

** Possibly not an AGB star.

${ }^{1}$ See applicable references in Table 5. 
D. A. García-Hernández et al.: Li and Zr in massive Galactic O-rich AGBs, Online Material p 6

Table 7. Galactic O-rich AGB stars for which no analysis of the lithium line was possible.

\begin{tabular}{lcccccc}
\hline \hline & & & & & & \\
IRAS name & Run & $\begin{array}{c}v_{\text {exp }}(\mathrm{OH}) \\
\left(\mathrm{km} \mathrm{s}^{-1}\right)\end{array}$ & Ref. $^{1}$ & $\begin{array}{c}\text { Period } \\
\text { (days) }\end{array}$ & Ref. $^{1}$ & Notes \\
\hline $01037+1219$ & 1,3 & 18.2 & 2 & 660 & 2 & $\mathrm{R}$ \\
$01304+6211$ & 3 & 11.0 & 2 & 1994 & 7 & $\ldots$ \\
$02316+6455$ & 3 & 15.5 & 7 & 534 & 3 & $\mathrm{R}$ \\
$05073+5248$ & 3 & 16.9 & 2 & 635 & 1 & $\mathrm{R}$ \\
$06297+4045$ & 3 & 12.0 & 2 & 520 & 10 & $\mathrm{R}$ \\
$09194-4518$ & 4 & 11.3 & 1 & $\ldots$ & $\ldots$ & $\mathrm{R}$ \\
$12384-4536$ & 4 & 13.2 & 1 & $\ldots$ & $\ldots$ & $\mathrm{R}$ \\
$13203-5536$ & 4 & 12.7 & 1 & $\ldots$ & $\ldots$ & $\ldots$ \\
$13328-6244$ & 4 & 25.1 & 1 & $\ldots$ & $\ldots$ & $\ldots$ \\
$13341-6246$ & 4 & 16.7 & 1 & $\ldots$ & $\ldots$ & $\mathrm{R}$ \\
$13517-6515$ & 4 & 17.9 & 1 & $\ldots$ & $\ldots$ & $\ldots$ \\
$15099-5509$ & 4 & 18.8 & 1 & $\ldots$ & $\ldots$ & $\mathrm{R}$ \\
$15303-5456$ & 4 & 24.0 & 1 & $\ldots$ & $\ldots$ & $\ldots$ \\
$17103-0559$ & 2,3 & 15.3 & 1 & 420 & 10 & $\ldots$ \\
$17239-2812$ & 3 & 20.7 & 1 & $\ldots$ & $\ldots$ & $\ldots$ \\
$17433-1750$ & 3 & 15.0 & 1 & $\ldots$ & $\ldots$ & $\mathrm{R}$ \\
$17433-2523$ & 3 & 19.9 & 1 & $\ldots$ & $\ldots$ & $\mathrm{R}$ \\
$17443-2519$ & 3 & 16.1 & 1 & $\ldots$ & $\ldots$ & $\ldots$ \\
$17501-2656$ & 3 & 23.0 & 1 & 928 & 8 & $\mathrm{R}$ \\
$18071-1727$ & 3 & 11.0 & 2 & 1488 & 9 & $\ldots$ \\
$18083-2630$ & 2,3 & 18.2 & 2 & $\ldots$ & $\ldots$ & $\ldots$ \\
$18172-2305$ & 3 & 15.9 & 1 & $\ldots$ & $\ldots$ & $\ldots$ \\
$18198-1249$ & 3 & 15.3 & 2 & 845 & 9 & $\ldots$ \\
$18257-1000$ & 2,3 & 19.1 & 2 & 1975 & 9 & $\ldots$ \\
$18273-0738$ & 2 & 14.3 & 2 & 700 & 5 & $\mathrm{R}$ \\
$18276-1431$ & 2 & 12.0 & 2 & 890 & 9 & $\mathrm{R}$ \\
$18312-1209$ & 3 & 12.3 & 1 & $\ldots$ & $\ldots$ & $\ldots$ \\
$18314-1131^{*}$ & 3 & $\ldots$ & 1 & $\ldots$ & $\ldots$ & $\mathrm{R}$ \\
$18348-0526$ & 2,3 & 13.9 & 2 & 1575 & 3 & $\ldots$ \\
$18432-0149$ & 2,3 & 17.5 & 2 & 1140 & 5 & $\ldots$ \\
$18437-0643$ & 2,3 & 12.1 & 2 & 652 & 9 & $\ldots$ \\
$18460-0254$ & 2,3 & 20.2 & 2 & 1730 & 5 & $\ldots$ \\
$18488-0107$ & 3 & 20.5 & 1 & 1540 & 4 & $\ldots$ \\
$18549+0208$ & 3 & 13.7 & 1 & 840 & 9 & $\ldots$ \\
$18560+0638$ & $1,2,3$ & 16.0 & 2 & 1033 & 5 & $\ldots$ \\
$19059-2219$ & 2,3 & 13.3 & 2 & 510 & 1 & $\mathrm{R}$ \\
$19161+2343$ & 2,3 & 17.9 & 2 & $\ldots$ & $\ldots$ & $\ldots$ \\
$19192+0922$ & 2,3 & 16.5 & 2 & 552 & 9 & $\ldots$ \\
$19254+1631$ & 2 & 19.0 & 2 & 1162 & 9 & $\mathrm{R}$ \\
$19426+4342$ & 1,3 & 8.6 & 1 & $\ldots$ & $\ldots$ & $\mathrm{R}$ \\
$20077-0625$ & 1,3 & 12.3 & 2 & 680 & 1 & $\mathrm{R}$ \\
$20109+3205$ & 3 & 5.7 & 1 & 382 & 1 & $\ldots$ \\
$20181+2234$ & 1,3 & 11.2 & 2 & $\ldots$ & $\ldots$ & $\ldots$ \\
$20272+3535$ & 3 & 12.0 & 2 & 1603 & 9 & $\ldots$ \\
$22177+5936$ & 3 & 15.1 & 2 & 1215 & 9 & $\ldots$ \\
\hline
\end{tabular}

* Single peak $\mathrm{OH}$ maser.

$\mathrm{R}$ : optical counterpart too red;

...: optical counterpart not found.

${ }^{1}$ See applicable references in Table 5.
Table 8. Comparison stars.

\begin{tabular}{lccccc}
\hline \hline IRAS name & Run & $\begin{array}{c}v_{\text {exp }}(\mathrm{OH}) \\
\left(\mathrm{km} \mathrm{s}^{-1}\right)\end{array}$ & Ref. $^{1}$ & $\begin{array}{c}\text { Period } \\
(\text { days })\end{array}$ & Ref. $^{1}$ \\
\hline $00213+3817$ & 1 & 9.0 & 10 & 409 & 1 \\
$02192+5821$ & 1 & 14.5 & 2 & 822 & 1 \\
$10436-3459$ & 4 & 11.3 & 1 & 104 & 1 \\
$18397+1738$ & 2 & 13.4 & 8 & 511 & 3 \\
$19133-1703$ & 1 & 10 & 10 & 395 & 1 \\
$20166+3717$ & 3 & $\ldots$ & $\ldots$ & 410 & 1 \\
$23416+6130$ & 1 & 26.5 & 2 & 925 & 1 \\
$23554+5612$ & 1 & 15.5 & 10 & 477 & 1 \\
$23587+6004$ & 1 & $\ldots$ & $\ldots$ & 186 & 1 \\
\hline
\end{tabular}

${ }^{1}$ See applicable references in Table 5.

Table 9. Peculiar C-rich AGB stars.

\begin{tabular}{lcccccc}
\hline \hline IRAS name & Run & $\begin{array}{c}v_{\text {exp }}(\mathrm{CO}) \\
\left(\mathrm{km} \mathrm{s}^{-1}\right)\end{array}$ & Ref. $^{1}$ & $\begin{array}{c}\text { Period } \\
(\text { days })\end{array}$ & Ref. $^{1}$ & Notes \\
\hline $00247+6922$ & 3 & 17.2 & 8 & 650 & 3 & $\ldots$ \\
$04130+3918$ & 3 & $\ldots$ & $\ldots$ & 470 & 10 & $\mathrm{~B}$ \\
$09425-6040$ & 4 & $\ldots$ & $\ldots$ & $\ldots$ & $\ldots$ & $\mathrm{B}$ \\
$17297+1747$ & 2 & 15.2 & 11 & 520 & 3 & $\mathrm{R}$ \\
$19321+2757$ & 2 & 24.4 & 8 & 625 & 3 & $\mathrm{R}$ \\
$19594+4047$ & 3 & 20.5 & 8 & 783 & 3 & $\ldots$ \\
$20072+3116$ & 2 & 25.6 & 8 & 550 & 3 & $\mathrm{R}$ \\
$23166+1655$ & 3 & 15.1 & 8 & 520 & 3 & $\ldots$ \\
$23320+4316$ & 3 & 14.7 & 8 & 620 & 3 & $\mathrm{R}$ \\
\hline
\end{tabular}

B: bright optical counterpart;

R: optical counterpart too red;

... optical counterpart not found.

${ }^{i}$ See applicable references in Table 5. 